ALEA, Lat. Am. J. Probab. Math. Stat. 15, 775-810 (2018)

DOI: 10.30757/ALEA.v15-29

\title{
Almost sure approximations in Hölder norms of a general stochastic process defined by a Young integral
}

\author{
Antoine Ayache, Céline Esser and Qidi Peng \\ UMR CNRS 8524 Laboratoire Paul Painlevé, \\ Université de Lille, \\ Bât. M2, Cité Scientifique, 59655 Villeneuve d'Ascq Cedex, France. \\ E-mail address: Antoine.Ayache@univ-lille.fr \\ Département de Mathématique, \\ Université de Liège, \\ Quartier Polytech 1, Bât. B37, Allée de la Découverte 12, \\ 4000 Liège, Belgium. \\ E-mail address: celine.esser@uliege.be \\ Institute of Mathematical Sciences, \\ Claremont Graduate University, \\ 710 N College Ave., \\ Claremont, California 91711, USA. \\ E-mail address: Qidi.Peng@cgu.edu
}

\begin{abstract}
We focus on a stochastic process $\{Y(t)\}_{t \in[0, v]}$ defined by a pathwise Young integral of a general form. Thanks to the Haar basis, we connect the classical method of approximation of $\{Y(t)\}_{t \in[0, v]}$ through Euler scheme and RiemannStieltjes sums with a new approach consisting in the use of an appropriate series representation of $\{Y(t)\}_{t \in[0, v]}$. This representation is obtained through a general compactly supported orthonormal wavelet basis. An advantage offered by the new approach with respect to the classical one is that a better almost sure rate of convergence in Hölder norms can be derived, under a general Wiener chaos condition. Also, this improved rate turns out to be optimal in some situations; typically, when the integrand and integrator associated to $\{Y(t)\}_{t \in[0, v]}$ are independent fractional Brownian motions with appropriate Hurst parameters.
\end{abstract}

Received by the editors October 6th, 2016; accepted May 19th, 2018.

2010 Mathematics Subject Classification. 60H05, 42C40, 60G17.

Key words and phrases. Stochastic integrals, Riemann-Stieltjes sums, Haar basis, wavelet bases, Hölder spaces.

This work has been partially supported by the Labex CEMPI (ANR-11-LABX-0007-01), the F.R.S.- FNRS and the GDR 3475 (Analyse Multifractale). 


\section{Introduction and motivations}

Throughout the article the integer $v \geq 1$ is arbitrary and fixed, and the interval $[0, v]$ is often denoted by $I$. We focus on a real-valued stochastic process $\{Y(t)\}_{t \in I}$ of a general form: it is given by the pathwise Young integral

$$
Y(t):=\int_{0}^{t} \sigma(s) \mathrm{d} X(s), \quad \text { for each } t \in I .
$$

Such kind of process is closely connected to (stochastic) differential equations driven by fractional Brownian motions and more generally by (random) Hölder functions with correlated increments (see e.g. Baudoin and Coutin, 2007; Decreusefond and Üstünel, 1999; Gubinelli et al., 2016, 2006; Lejay, 2010; Lyons et al., 2007; Nualart and Răşcanu, 2002; Ruzmaikina, 2000; Zähle, 1998, 2001). Therefore it is useful to find approximation procedures for $\{Y(t)\}_{t \in I}$ paths which converge at the fastest possible rate.

In this article, for convenience, the real-valued integrand $\sigma$ and integrator $X$ are assumed to be defined on the whole real line and not only on the interval $I:=[0, v]$. Also it is assumed that the paths of $\sigma$ and $X$, on any compact interval $\mathcal{K} \subset \mathbb{R}$, respectively belong to some Hölder spaces $C^{\alpha}(\mathcal{K})$ and $C^{\beta}(\mathcal{K})$, where $\alpha \in(0,1)$ and $\beta \in(0,1)$ do not depend on $\mathcal{K}$ and satisfy $\alpha+\beta>1$. Notice that this is a classical condition (see e.g. Lyons et al., 2007) which guarantees the existence of the pathwise Young integral in (1.1). We recall that, for any $\theta \in[0,1)$, the Hölder space $C^{\theta}(\mathcal{K})$ is defined as the Banach space of the continuous functions $h$, from $\mathcal{K}$ to $\mathbb{R}$, such that

$$
\sup _{\left(x_{1}, x_{2}\right) \in \mathcal{K}^{2}, x_{1}<x_{2}}\left\{\frac{\left|h\left(x_{1}\right)-h\left(x_{2}\right)\right|}{\left|x_{1}-x_{2}\right|^{\theta}}\right\}<+\infty ;
$$

it is equipped with the norm

$$
\|h\|_{C^{\theta}(\mathcal{K})}:=\|h\|_{\mathcal{K}, \infty}+\sup _{\left(x_{1}, x_{2}\right) \in \mathcal{K}^{2}, x_{1}<x_{2}}\left\{\frac{\left|h\left(x_{1}\right)-h\left(x_{2}\right)\right|}{\left|x_{1}-x_{2}\right|^{\theta}}\right\},
$$

where

$$
\|h\|_{\mathcal{K}, \infty}:=\sup _{x \in \mathcal{K}}|h(x)|
$$

denotes the uniform norm over $\mathcal{K}$. It is clear that $C^{0}(\mathcal{K})$ is nothing else than the whole space of the real-valued continuous functions on $\mathcal{K}$; moreover $\|\cdot\|_{C^{0}(\mathcal{K})}$ and $\|\cdot\|_{\mathcal{K}, \infty}$ are two equivalent norms. We mention that sometimes we will say that " $h$ satisfies a Hölder condition of order $\theta$ on $\mathcal{K}$ ", or more simply that " $h$ is $\theta$-Hölder continuous on $\mathcal{K}^{\prime}$ ", in order to indicate that $h$ belongs to the Hölder space $C^{\theta}(\mathcal{K})$.

The classical Euler scheme corresponding to Riemann-Stieltjes sums associated with dyadic intervals of fixed length $2^{-J}, J \in \mathbb{N}$, provides a natural method for approximating paths of the process $\{Y(t)\}_{t \in I}$. More precisely, for every dyadic number $m / 2^{J}$, with $m \in\left\{1, \ldots, 2^{J} v\right\}$, one approximates

$$
Y\left(\frac{m}{2^{J}}\right)=\int_{0}^{\frac{m}{2^{J}}} \sigma(s) \mathrm{d} X(s)=\sum_{l=0}^{m-1} \int_{\frac{l}{2^{J}}}^{\frac{l+1}{2^{J}}} \sigma(s) \mathrm{d} X(s)
$$

by

$$
Y_{J}\left(\frac{m}{2^{J}}\right):=\sum_{l=0}^{m-1} \sigma\left(\tilde{s}_{J, l}\right) \Delta_{J, l}(X),
$$


where the $\tilde{s}_{J, l}$ 's denote arbitrary fixed real numbers in the dyadic intervals $\left[l / 2^{J},(l+\right.$ $\left.1) / 2^{J}\right]$, and the $\Delta_{J, l}(X)$ 's are the first order increments of $X$ associated with these intervals, namely

$$
\Delta_{J, l}(X):=X\left(\frac{l+1}{2^{J}}\right)-X\left(\frac{l}{2^{J}}\right) .
$$

Then, using linear interpolation, one gets a random function, from $I$ to $\mathbb{R}, t \mapsto$ $Y_{J}^{R S}(t)$ which approximates the whole path $t \mapsto Y(t)$. More precisely, for every $t \in I$, one sets

$$
\begin{aligned}
Y_{J}^{R S}(t) & :=Y_{J}\left(\frac{\left[2^{J} t\right]}{2^{J}}\right)+\left(2^{J} t-\left[2^{J} t\right]\right)\left(Y_{J}\left(\frac{\left[2^{J} t\right]+1}{2^{J}}\right)-Y_{J}\left(\frac{\left[2^{J} t\right]}{2^{J}}\right)\right) \\
& =Y_{J}\left(\frac{\left[2^{J} t\right]}{2^{J}}\right)+\left(2^{J} t-\left[2^{J} t\right]\right) \sigma\left(\tilde{s}_{J,\left[2^{J} t\right]}\right) \Delta_{J,\left[2^{J} t\right]}(X)
\end{aligned}
$$

where $[\cdot]$ is the integer part function, and with the convention that

$$
Y_{J}^{R S}(0):=0 \quad \text { and } \quad Y_{J}^{R S}(v):=Y_{J}(v) .
$$

The following crucial proposition allows to derive a control on the uniform norm over $I$ of the error term $Y-Y_{J}^{R S}$.

Proposition 1.1 (Young - Loeve inequality). There exists a positive finite constant $\Lambda_{\alpha+\beta}$, depending only on $\alpha+\beta>1$, such that the inequality

$$
\begin{aligned}
& \left|\int_{t_{1}}^{t_{2}} \sigma(s) \mathrm{d} X(s)-\sigma\left(t_{1}\right)\left(X\left(t_{2}\right)-X\left(t_{1}\right)\right)\right| \\
& \quad \leq \Lambda_{\alpha+\beta}\|\sigma\|_{C^{\alpha}\left(\left[t_{1}, t_{2}\right]\right)}\|X\|_{C^{\beta}\left(\left[t_{1}, t_{2}\right]\right)}\left(t_{2}-t_{1}\right)^{\alpha+\beta}
\end{aligned}
$$

holds for all real numbers $t_{1}$ and $t_{2}$ satisfying $t_{1}<t_{2}$.

The proof of the proposition can be found in Section 1.3 of Lyons et al. (2007), just to give one reference.

Remark 1.2. It results from Proposition 1.1 and the Hölderianity assumption on the paths of $\sigma$ and $X$ that the paths of $Y$, on any compact interval $\mathcal{K} \subset \mathbb{R}$, belong to the Hölder space $C^{\beta}(\mathcal{K})$. In other words, one has $\|Y\|_{C^{\beta}(\mathcal{K})}<+\infty$.

Remark 1.3. It can easily be derived from Proposition 1.1 and (1.2) that the inequality

$$
\begin{aligned}
& \left|\int_{t_{1}}^{t_{2}} \sigma(s) \mathrm{d} X(s)-\sigma(\tilde{s})\left(X\left(t_{2}\right)-X\left(t_{1}\right)\right)\right| \\
& \leq\left(1+\Lambda_{\alpha+\beta}\right)\|\sigma\|_{C^{\alpha}\left(\left[t_{1}, t_{2}\right]\right)}\|X\|_{C^{\beta}\left(\left[t_{1}, t_{2}\right]\right)}\left(t_{2}-t_{1}\right)^{\alpha+\beta}
\end{aligned}
$$

holds for all real numbers $t_{1}, t_{2}$ and $\tilde{s}$ satisfying $t_{1}<t_{2}$ and $\tilde{s} \in\left[t_{1}, t_{2}\right]$.

In view of (1.1), (1.4), the triangle inequality and Remark 1.3, one has for all $m \in\left\{1, \ldots, 2^{J} v\right\}$

$$
\begin{aligned}
\left|Y\left(\frac{m}{2^{J}}\right)-Y_{J}\left(\frac{m}{2^{J}}\right)\right| & \leq \sum_{l=0}^{m-1}\left|\int_{l 2^{-J}}^{(l+1) 2^{-J}} \sigma(s) \mathrm{d} X(s)-\sigma\left(\tilde{s}_{J, l}\right) \Delta_{J, l}(X)\right| \\
& \leq c_{0} 2^{-J(\alpha+\beta-1)}
\end{aligned}
$$


where $c_{0}:=\left(1+\Lambda_{\alpha+\beta}\right)\|\sigma\|_{C^{\alpha}(I)}\|X\|_{C^{\beta}(I)}$. Then, the straightforward inequality

$$
\begin{aligned}
\left\|Y-Y_{J}^{R S}\right\|_{I, \infty} \leq\|Y\|_{C^{\beta}(I)} & 2^{-J \beta} \\
& +\max \left\{\left|Y\left(\frac{m}{2^{J}}\right)-Y_{J}\left(\frac{m}{2^{J}}\right)\right|, m \in\left\{1, \ldots, 2^{J} v\right\}\right\}
\end{aligned}
$$

implies that

$$
\left\|Y-Y_{J}^{R S}\right\|_{I, \infty} \leq c_{1} 2^{-J(\alpha+\beta-1)},
$$

where $c_{1}:=\|Y\|_{C^{\beta}(I)}+c_{0}$. The following proposition, proved in Appendix, provides an extension of (1.9) to some Hölder norms.

Proposition 1.4. There exists a random finite constant $c>0$ such that for all $\gamma \in[0, \beta)$ and $J \in \mathbb{N}$, one has

$$
\left\|Y-Y_{J}^{R S}\right\|_{C^{\gamma}(I)} \leq c 2^{-J \min (\beta-\gamma, \alpha+\beta-1)} .
$$

Is it possible to find approximation procedures, for $\{Y(t)\}_{t \in I}$ paths, allowing to have better rates of convergence than the one provided by (1.10)?

Studying this question is the main motivation behind our article; to this end, it is very useful to have an appropriate explicit expression for the error term.

The rest of the article is organized in the following way. In Section 2 we introduce, via a compactly supported orthonormal wavelet basis for $L^{2}(\mathbb{R})$, a series representation of the process $Y$; the partial sums of the series, denoted by $Y_{J}^{W}$, are somehow similar to the Riemann-Stieltjes sums $Y_{J}^{R S}$ and play the same role. Under the sole weak Hölderianity assumption, made at the beginning of the present section, one can only show that the approximation error $\left\|Y-Y_{J}^{W}\right\|_{C \gamma(I)}$ converges to 0 at the same rate as the one in (1.10). Yet, the wavelet approach offers the advantage to provide a rather "nice" explicit expression for the error term $Y-Y_{J}^{W}$. This advantage is exploited in Section 3 where it is further assumed that the Wiener chaos condition $(\mathcal{W} C)$, given by Definition 3.3, holds. Under this additional condition $(\mathcal{W} C)$, it turns out that the approximation error $\left\|Y-Y_{J}^{W}\right\|_{C^{\gamma}(I)}$ converges to 0 at a better rate of convergence than the one provided by (1.10). The main goal of Section 4 is to show that a wide class of integrators $X$ and integrands $\sigma$ satisfies the condition $(\mathcal{W} C)$; for example $X$ and $\sigma$ can be multiple Itô-Wiener integrals. Last but not least, the issue concerning the optimality of the improved rate of convergence is discussed in Section 5. This improved rate is optimal in some situations, such as when the integrand and integrator associated to $\{Y(t)\}_{t \in I}$ are independent fractional Brownian motions with appropriate Hurst parameters.

\section{The approximation method and its general rate of convergence}

In order to state the main result of this section, one first needs to introduce some notations. Assume that the collection of functions, from $\mathbb{R}$ to itself,

$$
\{\varphi(\bullet-l): l \in \mathbb{Z}\} \cup\left\{2^{j / 2} \psi\left(2^{j} \bullet-k\right):(j, k) \in \mathbb{Z}_{+} \times \mathbb{Z}\right\}
$$

satisfies one of the following two hypotheses.

$\left(\mathcal{H}_{1}\right)$ This collection is simply the Haar wavelet basis of $L^{2}(\mathbb{R})$, in other words one has $\varphi:=\mathbf{1}_{[0,1)}$ and $\psi:=\mathbf{1}_{[0,1 / 2)}-\mathbf{1}_{[1 / 2,1)}$. 
$\left(\mathcal{H}_{2}\right)$ This collection is an arbitrary compactly supported orthonormal wavelet basis of $L^{2}(\mathbb{R})$ such that the scaling function $\varphi$ and the mother wavelet $\psi$ are $\alpha$-Hölder continuous on $\mathbb{R}$, which means that

$$
\sup _{\left(x_{1}, x_{2}\right) \in \mathbb{R}^{2}, x_{1}<x_{2}}\left\{\frac{\left|\varphi\left(x_{1}\right)-\varphi\left(x_{2}\right)\right|+\left|\psi\left(x_{1}\right)-\psi\left(x_{2}\right)\right|}{\left|x_{1}-x_{2}\right|^{\alpha}}\right\}<+\infty .
$$

Notice that this order of Hölderianity $\alpha$ for $\varphi$ and $\psi$ is exactly the same as the one for the paths of the integrand $\sigma$ in (1.1).

It is known that (see e.g. Meyer, 1992; Daubechies, 1992; Wojtaszczyk, 1997) one has

$$
\int_{-\infty}^{+\infty} \varphi(x) \mathrm{d} x=1 \text { and } \int_{-\infty}^{+\infty} \psi(x) \mathrm{d} x=0,
$$

and that the integer translates of $\varphi$ form "a partition of unity" in the sense that:

$$
\sum_{l=-\infty}^{+\infty} \varphi(x-l)=1, \quad \text { for all } x \in \mathbb{R} .
$$

Now, notice that one can derive from the continuity of the paths of $\sigma$ that, for any fixed $t \in I$, the (random) function of the variable $s$

$$
s \mapsto \sigma_{t}(s):=\sigma(s) \mathbf{1}_{[0, t]}(s)
$$

belongs to $L^{2}(\mathbb{R})$. So, it can be expressed as the series of functions

$$
\sigma_{t}=\sum_{l=-\infty}^{+\infty} b_{0, l}(t) \varphi(\bullet-l)+\sum_{j=0}^{+\infty} \sum_{k=-\infty}^{+\infty} a_{j, k}(t) 2^{j / 2} \psi\left(2^{j} \bullet-k\right)
$$

which converges in $L^{2}(\mathbb{R})$. Notice that the coefficients of the series are defined as:

$$
b_{0, l}(t):=\int_{0}^{t} \sigma(s) \varphi(s-l) \mathrm{d} s
$$

and

$$
a_{j, k}(t):=2^{j / 2} \int_{0}^{t} \sigma(s) \psi\left(2^{j} s-k\right) \mathrm{d} s .
$$

Thus many of these coefficients vanish due to the compactness of the supports of $\varphi$ and $\psi$. More precisely, let $N_{1}$ and $N_{2}$ be two fixed integers with $N_{1}<N_{2}$, such that

$$
\operatorname{supp} \varphi \subseteq\left[N_{1}, N_{2}\right] \text { and } \operatorname{supp} \psi \subseteq\left[N_{1}, N_{2}\right] .
$$

Then, it is clear that

$$
\left(l+N_{1}, l+N_{2}\right) \cap(0, t)=\emptyset \Longrightarrow b_{0, l}(t)=0
$$

and

$$
\left(2^{-j}\left(k+N_{1}\right), 2^{-j}\left(k+N_{2}\right)\right) \cap(0, t)=\emptyset \Longrightarrow a_{j, k}(t)=0 .
$$

Thus, for any fixed $t \in I$, the equality (2.5) reduces to

$$
\sigma_{t}=\sum_{l=1-N_{2}}^{[t]-N_{1}} b_{0, l}(t) \varphi(\bullet-l)+\sum_{j=0}^{+\infty} \sum_{k=1-N_{2}}^{\left[2^{j} t\right]-N_{1}} a_{j, k}(t) 2^{j / 2} \psi\left(2^{j} \bullet-k\right)
$$


Next we consider, for any $J \in \mathbb{N}$, the partial sum

$$
\sigma_{t, J}:=\sum_{l=1-N_{2}}^{[t]-N_{1}} b_{0, l}(t) \varphi(\bullet-l)+\sum_{j=0}^{J-1} \sum_{k=1-N_{2}}^{\left[2^{j} t\right]-N_{1}} a_{j, k}(t) 2^{j / 2} \psi\left(2^{j} \bullet-k\right) .
$$

Using the multiresolution analysis (see e.g. Meyer, 1992; Daubechies, 1992; Wojtaszczyk, 1997) generated by $\varphi$, one can derive that

$$
\sigma_{t, J}=\sum_{l=1-N_{2}}^{\left[2^{J} t\right]-N_{1}} b_{J, l}(t) 2^{J / 2} \varphi\left(2^{J} \bullet-l\right)
$$

where

$$
b_{J, l}(t):=2^{J / 2} \int_{0}^{t} \sigma(s) \varphi\left(2^{J} s-l\right) \mathrm{d} s .
$$

In view of (2.8) and (2.10), the random functions $\sigma_{t, J}, t \in I$ and $J \in \mathbb{N}$, have compact supports uniformly bounded in $t$ and $J$. More precisely, setting

$$
Q_{1}:=1-\left(N_{2}-N_{1}\right) \quad \text { and } \quad Q_{2}:=v+\left(N_{2}-N_{1}\right),
$$

then one has supp $\sigma_{t, J} \subseteq\left[Q_{1}, Q_{2}\right]$, for every $t \in I$ and all $J \in \mathbb{N}$.

Definition 2.1. For any $t \in I$ and all $J \in \mathbb{N}$, one sets

$$
Y_{J}^{W}(t):=\int_{Q_{1}}^{Q_{2}} \sigma_{t, J}(s) \mathrm{d} X(s)
$$

It is important to note that, in view of (2.9) and (2.10), this pathwise Young integral satisfies the two equalities:

$$
Y_{J}^{W}(t)=\sum_{l=1-N_{2}}^{[t]-N_{1}} b_{0, l}(t) \eta_{0, l}+\sum_{j=0}^{J-1} \sum_{k=1-N_{2}}^{\left[2^{j} t\right]-N_{1}} a_{j, k}(t) \lambda_{j, k}=\sum_{l=1-N_{2}}^{\left[2^{J} t\right]-N_{1}} b_{J, l}(t) \eta_{J, l},
$$

where the stochastic processes $\left\{\lambda_{j, k}\right\}_{(j, k) \in \mathbb{Z}_{+} \times \mathbb{Z}}$ and $\left\{\eta_{J, l}\right\}_{(J, l) \in \mathbb{Z}_{+} \times \mathbb{Z}}$ are defined as

$$
\lambda_{j, k}:=2^{j / 2} \int_{2^{-j}\left(k+N_{1}\right)}^{2^{-j}\left(k+N_{2}\right)} \psi\left(2^{j} s-k\right) \mathrm{d} X(s), \quad \text { for all }(j, k) \in \mathbb{Z}_{+} \times \mathbb{Z},
$$

and

$$
\eta_{J, l}:=2^{J / 2} \int_{2^{-J}\left(l+N_{1}\right)}^{2^{-J}\left(l+N_{2}\right)} \varphi\left(2^{J} s-l\right) \mathrm{d} X(s), \quad \text { for all }(J, l) \in \mathbb{Z}_{+} \times \mathbb{Z} .
$$

Remark 2.2. The main result of the present section, that is the following theorem, shows that $\{Y(t)\}_{t \in I}$ paths can be approximated by $\left\{Y_{J}^{W}(t)\right\}_{t \in I}$ paths. Actually this approximation procedure can be connected to the one with Riemann-Stieltjes sums, presented in the previous section. More precisely, assume that the basis in (2.1) is the Haar wavelet basis, and that the $\tilde{s}_{J, l}$ 's in (1.4) are chosen so that

$$
\sigma\left(\tilde{s}_{J, l}\right)=2^{J} \int_{2^{-J} l}^{2^{-J}(l+1)} \sigma(s) \mathrm{d} s, \quad \text { for every } l \in\left\{0, \ldots, 2^{J} v-1\right\} .
$$

Then, in view of (1.6), (1.4), (2.13), (2.11) and (2.15), one has

$$
Y_{J}^{W}\left(2^{-J} l\right)=Y_{J}^{R S}\left(2^{-J} l\right), \quad \text { for all } l \in\left\{0, \ldots, 2^{J} v\right\} \text {. }
$$


Theorem 2.3. There is a finite random constant $c>0$ such that, for all $\gamma \in[0, \beta)$ and $J \in \mathbb{N}$, one has

$$
\left\|Y-Y_{J}^{W}\right\|_{C^{\gamma}(I)} \leq c 2^{-J \min (\beta-\gamma, \alpha+\beta-1)} .
$$

A straightforward but important consequence of this theorem and the first equality in (2.13) is the following:

Corollary 2.4. Almost every path of the process $\{Y(t)\}_{t \in I}$ can be expressed as path of the following series of random functions:

$$
\sum_{l=1-N_{2}}^{[\bullet]-N_{1}} b_{0, l}(\bullet) \eta_{0, l}+\sum_{j=0}^{+\infty} \sum_{k=1-N_{2}}^{\left[2^{j} \bullet\right]-N_{1}} a_{j, k}(\bullet) \lambda_{j, k},
$$

which converges in the Hölder space $C^{\gamma}(I)$, for any $\gamma \in[0, \beta)$.

Remark 2.5. We mention that, in some sense, Corollary 2.4 is reminiscent of Theorem 3.14 in Gubinelli et al. (2016) which provides, via the Haar basis, a series representation for processes defined by Young integrals. Also, Corollary 2.4 might have some connections with the pathwise stochastic integral introduced in Ciesielski et al. (1993) via the same basis. Yet, we point out that there is a considerable difference between the approach in the two articles Ciesielski et al. (1993); Gubinelli et al. (2016) and the one in our present paper. Indeed, in these two articles it is the "derivative" of the integrator, namely $\mathrm{d} X(\bullet)$, which is expanded in the Haar basis in order to get the series representation for the integral, while in our present paper it is the integrand $\sigma(\bullet) \mathbf{1}_{[0, t]}(\bullet)$ which is expanded in this basis, and more generally in a wavelet basis.

In order to prove Theorem 2.3, one needs to introduce, for each fixed $J \in \mathbb{N}$ and $t_{1}, t_{2} \in I$ satisfying $t_{1}<t_{2}$, the following two finite sets of indices $l$ :

$$
\mathbb{L}_{J, t_{1}, t_{2}}:=\left\{l \in\left\{1-N_{2}, \ldots, 2^{J} v-N_{1}\right\}:\left[\frac{l+N_{1}}{2^{J}}, \frac{l+N_{2}}{2^{J}}\right] \subseteq\left[t_{1}, t_{2}\right]\right\},
$$

and

$$
\begin{array}{r}
\partial \mathbb{L}_{J, t_{1}, t_{2}}:=\left\{l \in\left\{1-N_{2}, \ldots, 2^{J} v-N_{1}\right\}: l \notin \mathbb{L}_{J, t_{1}, t_{2}}\right. \\
\left.\quad \text { and }\left[\mu_{J, l}\left(t_{1}, t_{2}\right), \nu_{J, l}\left(t_{1}, t_{2}\right)\right] \neq \emptyset\right\}
\end{array}
$$

where, for every $l \in \partial \mathbb{L}_{J, t_{1}, t_{2}}$, the interval $\left[\mu_{J, l}\left(t_{1}, t_{2}\right), \nu_{J, l}\left(t_{1}, t_{2}\right)\right]$ is defined as

$$
\left[\mu_{J, l}\left(t_{1}, t_{2}\right), \nu_{J, l}\left(t_{1}, t_{2}\right)\right]:=\left[\frac{l+N_{1}}{2^{J}}, \frac{l+N_{2}}{2^{J}}\right] \cap\left[t_{1}, t_{2}\right] .
$$

Notice that $\mathbb{L}_{J, t_{1}, t_{2}}$ or $\partial \mathbb{L}_{J, t_{1}, t_{2}}$ can sometime be the empty set.

Also, the proof of Theorem 2.3 requires to make use of the three properties $\left(\mathcal{P}_{1}\right)$, $\left(\mathcal{P}_{2}\right)$ and $\left(\mathcal{P}_{3}\right)$ of the scaling function $\varphi$ listed in the following lemma.

Lemma 2.6. Under the hypothesis $\left(\mathcal{H}_{1}\right)$ or the hypothesis $\left(\mathcal{H}_{2}\right)$, given at the very beginning of the present section, the following four results hold.

$\left(\mathcal{P}_{1}\right)$ There is a finite random constant $c_{1}>0$ such that, for every $J \in \mathbb{N}$ and every $l \in\left\{1-N_{2}, \ldots, 2^{J} v-N_{1}\right\}$, one has

$$
\left|\int_{2^{-J}\left(l+N_{1}\right)}^{2^{-J}\left(l+N_{2}\right)} \varphi\left(2^{J} s-l\right) \mathrm{d} X(s)\right| \leq c_{1} 2^{-J \beta} .
$$


$\left(\mathcal{P}_{1}^{\prime}\right)$ There is a finite random constant $c_{1}^{\prime}>0$ such that, for every $j \in \mathbb{Z}_{+}$and every $k \in\left\{1-N_{2}, \ldots, 2^{j} v-N_{1}\right\}$, one has

$$
\left|\int_{2^{-j}\left(k+N_{1}\right)}^{2^{-j}\left(k+N_{2}\right)} \psi\left(2^{j} s-k\right) \mathrm{d} X(s)\right| \leq c_{1}^{\prime} 2^{-j \beta} .
$$

$\left(\mathcal{P}_{2}\right)$ There is a finite random constant $c_{2}>0$ such that, for every $J \in \mathbb{N}$ and every $l \in\left\{1-N_{2}, \ldots, 2^{J} v-N_{1}\right\}$, one has

$$
\left|\int_{2^{-J}\left(l+N_{1}\right)}^{2^{-J}\left(l+N_{2}\right)}\left(\sigma(s)-\bar{\sigma}_{J, l}\right) \varphi\left(2^{J} s-l\right) \mathrm{d} X(s)\right| \leq c_{2} 2^{-J(\alpha+\beta)}
$$

where

$$
\bar{\sigma}_{J, l}:=2^{J} \int_{2^{-J}\left(l+N_{1}\right)}^{2^{-J}\left(l+N_{2}\right)} \sigma(s) \varphi\left(2^{J} s-l\right) \mathrm{d} s .
$$

$\left(\mathcal{P}_{3}\right)$ There is a finite random constant $c_{3}>0$ such that, for every $t_{1}, t_{2} \in I$ with $t_{1}<t_{2}$, every $J \in \mathbb{N}$ and every $l \in \partial \mathbb{L}_{J, t_{1}, t_{2}}$ (see (2.17)), one has

$$
\left|\int_{t_{1}}^{t_{2}} \sigma(s) \varphi\left(2^{J} s-l\right) \mathrm{d} X(s)\right| \leq c_{3} \min \left(2^{-J \beta},\left|t_{1}-t_{2}\right|^{\beta}\right) .
$$

The proof of Lemma 2.6 is postponed to Appendix, we now focus on that of Theorem 2.3.

Proof of Theorem 2.3: In view of (1.2), it is enough to show that there is a finite random constant $c>0$ such that, for all $\gamma \in[0, \beta)$, all $t_{1}, t_{2} \in I$ with $t_{1}<t_{2}$, and all $J \in \mathbb{N}$, one has

$$
\frac{\left|Y\left(t_{2}\right)-Y\left(t_{1}\right)-Y_{J}^{W}\left(t_{2}\right)+Y_{J}^{W}\left(t_{1}\right)\right|}{\left|t_{1}-t_{2}\right|^{\gamma}} \leq c 2^{-J \min (\beta-\gamma, \alpha+\beta-1)} .
$$

Notice that (2.13), (2.11), the first inclusion in (2.8), (2.16), (2.22), (2.15), (2.17), and (2.18) imply that

$$
\begin{gathered}
Y_{J}^{W}\left(t_{2}\right)-Y_{J}^{W}\left(t_{1}\right)=\sum_{l \in \mathbb{L}_{J, t_{1}, t_{2}}} \int_{2^{-J}\left(l+N_{1}\right)}^{2^{-J}\left(l+N_{2}\right)} \bar{\sigma}_{J, l} \varphi\left(2^{J} s-l\right) \mathrm{d} X(s) \\
+\sum_{l \in \partial \mathbb{L}_{J, t_{1}, t_{2}}} \eta_{J, l} 2^{J / 2} \int_{t_{1}}^{t_{2}} \sigma(s) \varphi\left(2^{J} s-l\right) \mathrm{d} s .
\end{gathered}
$$

Moreover, it follows from (1.1), (2.3), the first inclusion in (2.8), (2.16), (2.17), and (2.18) that

$$
\begin{gathered}
Y\left(t_{2}\right)-Y\left(t_{1}\right)=\int_{t_{1}}^{t_{2}} \sigma(s) \mathrm{d} X(s)=\int_{t_{1}}^{t_{2}} \sigma(s)\left(\sum_{l=-\infty}^{+\infty} \varphi\left(2^{J} s-l\right)\right) \mathrm{d} X(s) \\
=\int_{t_{1}}^{t_{2}} \sigma(s)\left(\sum_{l \in \mathbb{L}_{J, t_{1}, t_{2}}} \varphi\left(2^{J} s-l\right)+\sum_{l \in \partial \mathbb{L}_{J, t_{1}, t_{2}}} \varphi\left(2^{J} s-l\right)\right) \mathrm{d} X(s) \\
=\sum_{l \in \mathbb{L}_{J, t_{1}, t_{2}}} \int_{2^{-J}\left(l+N_{1}\right)}^{2^{-J}\left(l+N_{2}\right)} \sigma(s) \varphi\left(2^{J} s-l\right) \mathrm{d} X(s) \\
+\sum_{l \in \partial \mathbb{L}_{J, t_{1}, t_{2}}} \int_{t_{1}}^{t_{2}} \sigma(s) \varphi\left(2^{J} s-l\right) \mathrm{d} X(s) .
\end{gathered}
$$


Next using (2.25), (2.26) and the triangle inequality, one gets

$$
\left|Y\left(t_{2}\right)-Y\left(t_{1}\right)-Y_{J}^{W}\left(t_{2}\right)+Y_{J}^{W}\left(t_{1}\right)\right| \leq \mathcal{A}_{J}^{(1)}\left(t_{1}, t_{2}\right)+\mathcal{A}_{J}^{(2)}\left(t_{1}, t_{2}\right),
$$

where

$$
\mathcal{A}_{J}^{(1)}\left(t_{1}, t_{2}\right):=\sum_{l \in \mathbb{L}_{J, t_{1}, t_{2}}}\left|\int_{2^{-J}\left(l+N_{1}\right)}^{2^{-J}\left(l+N_{2}\right)}\left(\sigma(s)-\bar{\sigma}_{J, l}\right) \varphi\left(2^{J} s-l\right) \mathrm{d} X(s)\right|
$$

and

$$
\begin{aligned}
\mathcal{A}_{J}^{(2)}\left(t_{1}, t_{2}\right):=\sum_{l \in \partial \mathbb{L}_{J, t_{1}, t_{2}}} \mid \int_{t_{1}}^{t_{2}} \sigma(s) \varphi\left(2^{J} s-l\right) \mathrm{d} X(s) & \\
& \quad-\eta_{J, l} 2^{J / 2} \int_{t_{1}}^{t_{2}} \sigma(s) \varphi\left(2^{J} s-l\right) \mathrm{d} s .
\end{aligned}
$$

Let us first provide an appropriate upper bound for $\mathcal{A}_{J}^{(1)}\left(t_{1}, t_{2}\right)$. Assume that $l \in \mathbb{L}_{J, t_{1}, t_{2}}$ is arbitrary. Using $\left(\mathcal{P}_{2}\right)$ in Lemma 2.6, one gets

$$
\left|\int_{2^{-J}\left(l+N_{1}\right)}^{2^{-J}\left(l+N_{2}\right)}\left(\sigma(s)-\bar{\sigma}_{J, l}\right) \varphi\left(2^{J} s-l\right) \mathrm{d} X(s)\right| \leq c_{1} 2^{-J(\alpha+\beta)}
$$

where the finite random constant $c_{1}>0$ does not depend on $J, t_{1}, t_{2}$ and $l$; in fact $c_{1}$ is nothing else than the finite random constant $c_{2}$ in (2.21). Also notice that, in view of (2.16), there is a finite deterministic constant $c_{2}>0$ (which should not be confused with the constant $c_{2}$ in (2.21)) not depending on $J, t_{1}$ and $t_{2}$, such that, for all $J \in \mathbb{N}$, one has

$$
\operatorname{card}\left(\mathbb{L}_{J, t_{1}, t_{2}}\right) \leq c_{2} 2^{J}\left|t_{1}-t_{2}\right| .
$$

Thus, (2.28), (2.30) and (2.31) yield

$$
\mathcal{A}_{J}^{(1)}\left(t_{1}, t_{2}\right) \leq c_{3}\left|t_{1}-t_{2}\right| 2^{-J(\alpha+\beta-1)}
$$

where $c_{3}>0$ is a finite random constant not depending on $J, t_{1}$ and $t_{2}$.

Let us now give an appropriate upper bound for $\mathcal{A}_{J}^{(2)}\left(t_{1}, t_{2}\right)$. It follows from (2.29) and the triangle inequality that

$$
\mathcal{A}_{J}^{(2)}\left(t_{1}, t_{2}\right) \leq \sum_{l \in \partial \mathbb{L}_{J, t_{1}, t_{2}}}\left(\mathcal{B}_{J, l}^{(1)}\left(t_{1}, t_{2}\right)+\mathcal{B}_{J, l}^{(2)}\left(t_{1}, t_{2}\right)\right)
$$

where, for every $l \in \partial \mathbb{L}_{J, t_{1}, t_{2}}$,

$$
\mathcal{B}_{J, l}^{(1)}\left(t_{1}, t_{2}\right):=\left|\eta_{J, l} 2^{J / 2} \int_{t_{1}}^{t_{2}} \sigma(s) \varphi\left(2^{J} s-l\right) \mathrm{d} s\right|
$$

and

$$
\mathcal{B}_{J, l}^{(2)}\left(t_{1}, t_{2}\right):=\left|\int_{t_{1}}^{t_{2}} \sigma(s) \varphi\left(2^{J} s-l\right) \mathrm{d} X(s)\right| .
$$

First, one provides an appropriate upper bound for $\mathcal{B}_{J, l}^{(1)}\left(t_{1}, t_{2}\right)$. Using $(2.15)$ and $\left(\mathcal{P}_{1}\right)$ in Lemma 2.6, one obtains that

$$
\left|\eta_{J, l}\right| \leq c_{4} 2^{-J(\beta-1 / 2)},
$$


where $c_{4}$ is nothing else than the finite random constant $c_{1}$ in (2.19), which does not depend on $J, t_{1}, t_{2}$ and $l$. Moreover, it can easily be derived from (1.3), (2.17), (2.18) and (2.8) that

$$
\begin{aligned}
& \left|\int_{t_{1}}^{t_{2}} \sigma(s) \varphi\left(2^{J} s-l\right) \mathrm{d} s\right| \\
& \quad \leq\|\sigma\|_{\left[Q_{1}, Q_{2}\right], \infty}\|\varphi\|_{\left[N_{1}, N_{2}\right], \infty}\left(N_{2}-N_{1}\right) \min \left(2^{-J},\left|t_{1}-t_{2}\right|\right) .
\end{aligned}
$$

Putting together (2.34), (2.36) and (2.37) leads to

$$
\mathcal{B}_{J, l}^{(1)}\left(t_{1}, t_{2}\right) \leq c_{5} 2^{J(1-\beta)} \min \left(2^{-J},\left|t_{1}-t_{2}\right|\right),
$$

with $c_{5}>0$ being a finite random constant, not depending on $J, t_{1}, t_{2}$ and $l$.

Now, one provides an appropriate upper bound for $\mathcal{B}_{J, l}^{(2)}\left(t_{1}, t_{2}\right)$. Using $\left(\mathcal{P}_{3}\right)$ in Lemma 2.6, one obtains

$$
\mathcal{B}_{J, l}^{(2)}\left(t_{1}, t_{2}\right) \leq c_{6} \min \left(2^{-J \beta},\left|t_{1}-t_{2}\right|^{\beta}\right),
$$

where $c_{6}$ is in fact the finite random constant $c_{3}$ in (2.23), that does not depend on $J, t_{1}, t_{2}$ and $l$. Next, one sets $c_{7}:=2\left(N_{2}-N_{1}+1\right)$. It can be derived from (2.17) and (2.18) that

$$
\operatorname{card}\left(\partial \mathbb{L}_{J, t_{1}, t_{2}}\right) \leq c_{7}
$$

Then, it follows from $(2.33),(2.38),(2.39)$ and (2.40) that

$$
\mathcal{A}_{J}^{(2)}\left(t_{1}, t_{2}\right) \leq c_{8}\left(\min \left(2^{-J \beta}, 2^{J(1-\beta)}\left|t_{1}-t_{2}\right|\right)+\min \left(2^{-J \beta},\left|t_{1}-t_{2}\right|^{\beta}\right)\right),
$$

where $c_{8}:=c_{7} \max \left(c_{5}, c_{6}\right)$. Next, one sets $c_{9}:=\max \left(c_{3}, c_{8}\right)$. Combining (2.27) with (2.32) and (2.41) yields

$$
\begin{aligned}
\mid Y\left(t_{2}\right)-Y\left(t_{1}\right)- & Y_{J}^{W}\left(t_{2}\right)+Y_{J}^{W}\left(t_{1}\right) \mid \leq c_{9}\left(\left|t_{1}-t_{2}\right| 2^{-J(\alpha+\beta-1)}\right. \\
& \left.+\min \left(2^{-J \beta}, 2^{J(1-\beta)}\left|t_{1}-t_{2}\right|\right)+\min \left(2^{-J \beta},\left|t_{1}-t_{2}\right|^{\beta}\right)\right) .
\end{aligned}
$$

Finally, in view of (2.42), a straightforward computation shows that, in both cases $\left|t_{1}-t_{2}\right| \geq 2^{-J}$ and $\left|t_{1}-t_{2}\right|<2^{-J}$, one has, for every $\gamma \in[0, \beta)$,

$$
\begin{aligned}
\frac{\left|Y\left(t_{2}\right)-Y\left(t_{1}\right)-Y_{J}^{W}\left(t_{2}\right)+Y_{J}^{W}\left(t_{1}\right)\right|}{\left|t_{1}-t_{2}\right|^{\gamma}} & \leq c_{9}\left(v^{1-\gamma} 2^{-J(\alpha+\beta-1)}+2^{-J(\beta-\gamma)+1}\right) \\
& \leq c 2^{-J \min (\beta-\gamma, \alpha+\beta-1)}
\end{aligned}
$$

where $c:=3 c_{9} v^{1-\gamma}$. Therefore (2.24) holds, hence the conclusion.

\section{A better rate of convergence under the Wiener chaos condition $(\mathcal{W} C)$}

First, it is useful to make some brief recalls on the notion of Wiener chaos; our presentation of it is inspired by the one in the book Janson (1997). Throughout this article the underlying probability space is denoted by $(\Omega, \mathcal{F}, \mathbb{P})$. Moreover, for any fixed $p \in(0,+\infty)$, the space of the real-valued random variables on $(\Omega, \mathcal{F}, \mathbb{P})$ having a finite absolute moment of order $p$ is denoted by $L^{p}(\Omega, \mathcal{F}, \mathbb{P})$. 
Definition 3.1 (Wiener chaos). Let $G$ be an arbitrary fixed Gaussian subspace of $L^{2}(\Omega, \mathcal{F}, \mathbb{P})$, that is a closed subspace consisting of real-valued centred Gaussian random variables. Let $n$ be a nonnegative integer, the Wiener chaos of order $n$ associated with $G$ is denoted by $\overline{\mathcal{P}}_{n}(G)$, or more simply by $\overline{\mathcal{P}}_{n}$. The space $\overline{\mathcal{P}}_{0}$ is defined to be the closed subspace of $L^{2}(\Omega, \mathcal{F}, \mathbb{P})$ consisting of all the constant random variables. When $n \geq 1$, the space $\overline{\mathcal{P}}_{n}$ is defined as the closed subspace of $L^{2}(\Omega, \mathcal{F}, \mathbb{P})$ spanned by the following set of random variables:

$$
\left\{\prod_{l=1}^{n} g_{l}^{m_{l}}:\left(g_{1}, \ldots, g_{n}\right) \in G^{n} \text { and }\left(m_{1}, \ldots, m_{n}\right) \in \mathbb{Z}_{+}^{n} \text { with } \sum_{l=1}^{n} m_{l} \leq n\right\} .
$$

Remarks 3.2 .

(a) One clearly has $\overline{\mathcal{P}}_{n} \subseteq \overline{\mathcal{P}}_{n+1}$, for every $n \in \mathbb{Z}_{+}$. Moreover, for all fixed $p \in$ $(0,+\infty)$, the space $\overline{\mathcal{P}}_{*}:=\bigcup_{n \in \mathbb{Z}_{+}} \overline{\mathcal{P}}_{n}$ is dense in $L^{p}(\Omega, \mathcal{F}(G), \mathbb{P})$, where $\mathcal{F}(G)$ denotes the smallest sub $\sigma$-field of $\mathcal{F}$ for which all the random variables in $G$ are measurable (see for instance Theorem 3.51 in Janson, 1997).

(b) The $L^{p}(\Omega)$-norms are equivalent on $\overline{\mathcal{P}}_{n}$ (see for instance Theorem 3.50 in Janson, 1997). In other words, there are two positive and finite universal constants $c_{1}(n, p) \leq c_{2}(n, p)$, depending only on $n$ and $p$, such that, for any random variable $\chi \in \overline{\mathcal{P}}_{n}$, one has

$$
c_{1}(n, p)\left(\mathbb{E}\left[|\chi|^{2}\right]\right)^{p / 2} \leq \mathbb{E}\left[|\chi|^{p}\right] \leq c_{2}(n, p)\left(\mathbb{E}\left[|\chi|^{2}\right]\right)^{p / 2} .
$$

(c) For every fixed $n \in \mathbb{Z}_{+}$and for each sequence of random variables in $\overline{\mathcal{P}}_{n}$, convergence in probability is equivalent to convergence in $L^{p}(\Omega)$-norm, for any fixed $p \in(0,+\infty)$ (see for instance Theorem 3.50 in Janson, 1997).

(d) For all fixed integer $n \geq 1$, there exists a positive finite universal constant $c_{3}(n)$, depending only on $n$, such that, for every random variable $\chi \in \overline{\mathcal{P}}_{n}$ and for each real number $y \geq 2$, one has

$$
\mathbb{P}\left(|\chi|>y\|\chi\|_{L^{2}(\Omega)}\right) \leq \exp \left(-c_{3}(n) y^{2 / n}\right),
$$

where $\|\chi\|_{L^{2}(\Omega)}:=\left(\mathbb{E}\left[|\chi|^{2}\right]\right)^{1 / 2}$ (see for instance Theorem 6.7 in Janson, 1997).

Let us now precisely define the so-called Wiener chaos condition $(\mathcal{W} C)$.

Definition 3.3 (the Wiener chaos condition $(\mathcal{W} C)$ ). One says that the stochastic process $\{Y(t)\}_{t \in I}$, defined through the pathwise Young integral (1.1), satisfies the Wiener chaos condition $(\mathcal{W} C)$ when, for some arbitrary integer $n \geq 1$, the integrand $\{\sigma(s)\}_{s \in \mathbb{R}}$ and the integrator $\{X(s)\}_{s \in \mathbb{R}}$ are two stochastic processes belonging to the Wiener chaos $\overline{\mathcal{P}}_{n}$ (i.e. $\sigma(s) \in \overline{\mathcal{P}}_{n}$ and $X(s) \in \overline{\mathcal{P}}_{n}$, for all $s \in \mathbb{R}$ ) and possessing the following two properties:

$\left(\mathcal{C}_{1}\right)$ There exist two positive real numbers $\alpha_{0}$ and $\beta_{0}$, satisfying $\alpha_{0} \leq 1, \beta_{0} \leq 1$ and $\alpha_{0}+\beta_{0}>1$, such that the restrictions of $\{\sigma(s)\}_{s \in \mathbb{R}}$ and $\{X(s)\}_{s \in \mathbb{R}}$ to any compact interval $\mathcal{K}$ are respectively $\alpha_{0^{-H o ̈ l d e r}}$ continuous and $\beta_{0^{-}}$ Hölder continuous in quadratic mean. In other words, the inequalities

$$
\mathbb{E}\left[\left|\sigma\left(s_{1}\right)-\sigma\left(s_{2}\right)\right|^{2}\right] \leq c_{0}\left|s_{1}-s_{2}\right|^{2 \alpha_{0}} \quad \text { for all } s_{1}, s_{2} \in \mathcal{K}
$$


and

$$
\mathbb{E}\left[\left|X\left(s_{1}\right)-X\left(s_{2}\right)\right|^{2}\right] \leq c_{1}\left|s_{1}-s_{2}\right|^{2 \beta_{0}} \quad \text { for all } s_{1}, s_{2} \in \mathcal{K}
$$

hold, for some finite deterministic constants $c_{0}$ and $c_{1}$ which a priori depend on $\mathcal{K}$.

$\left(\mathcal{C}_{2}\right)$ The wavelet coefficients ${ }^{1} a_{j, k}:=a_{j, k}(v)$ and $\lambda_{j, k}$, defined through (2.7) and (2.14), have the following "short-range dependence" property: the inequality

$$
\sum_{k_{1}=-N_{1}}^{2^{j} v-N_{2}} \sum_{k_{2}=-N_{1}}^{2^{j} v-N_{2}}\left|\mathbb{E}\left[a_{j, k_{1}} \lambda_{j, k_{1}} a_{j, k_{2}} \lambda_{j, k_{2}}\right]\right| \leq c_{2} 2^{-2 j\left(\alpha_{0}+\beta_{0}-1 / 2\right)}
$$

is satisfied, for some finite deterministic constant $c_{2}>0$ and for all positive integer $j$ such that $2^{j} v \geq N_{2}-N_{1}$. Recall that $N_{1}$ and $N_{2}$ are two fixed integers satisfying (2.8).

Remarks 3.4. Assume that the Wiener chaos condition $(\mathcal{W} C)$ holds.

(a) Combining $\left(\mathcal{C}_{1}\right)$ with the second inequality in (3.1), where taking $\chi=$ $\sigma\left(s_{1}\right)-\sigma\left(s_{2}\right)$ (resp. $\chi=X\left(s_{1}\right)-X\left(s_{2}\right)$ ) and $p$ large enough, and using the Kolmogorov-Čentsov Hölder continuity theorem (see Chapter 2 in Karatzas and Shreve, 1988), it can be shown that the process $\sigma$ (resp. $X$ ) has a modification whose paths belong to the Hölder spaces $C^{\alpha}(\mathcal{K})$, for all $\alpha \in\left(0, \alpha_{0}\right)$ (resp. $C^{\beta}(\mathcal{K})$, for all $\beta \in\left(0, \beta_{0}\right)$ ) and for all compact intervals $\mathcal{K}$. This modification is always identified with $\sigma$ (resp. $X$ ) itself, therefore Proposition 1.1 implies that the paths of the process $\{Y(t)\}_{t \in I}$ are Hölder continuous of any order $\beta \in\left(0, \beta_{0}\right)$.

(b) In view of the fact that for $s \in \mathbb{R}$, the random variables $\sigma(s)$ and $X(s)$ belong to the Wiener chaos $\overline{\mathcal{P}}_{n}$ (for some arbitrary integer $n \geq 1$ ), by approximating the pathwise Young integral in (1.1) by Riemann-Stieltjes sums and by using Remark $3.2(\mathrm{c})$, it can be shown that, for each $t \in I$, the random variable $Y(t)$ belongs to the Wiener chaos $\overline{\mathcal{P}}_{2 n}$.

(c) Using similar arguments, one can prove that for all $(J, l) \in \mathbb{Z}_{+} \times \mathbb{Z},(j, k) \in$ $\mathbb{Z}_{+} \times \mathbb{Z}$ and $t \in I$, the random variables $a_{j, k}(t), b_{J, l}(t), \lambda_{j, k}$ and $\eta_{J, l}$ (see (2.7), (2.11), (2.14) and (2.15)) belong to the Wiener chaos $\overline{\mathcal{P}}_{n}$. This implies that $Y_{J}^{W}(t)$ (see Definition 2.1) belongs to the Wiener chaos $\overline{\mathcal{P}}_{2 n}$.

The main goal of the present section is to obtain the following theorem.

Theorem 3.5. Under the condition $(\mathcal{W} C)$, for any fixed real numbers $\alpha \in\left(0, \alpha_{0}\right)$, $\beta \in\left(0, \beta_{0}\right)$ and $\gamma \geq 0$ satisfying $\alpha+\beta>1$ and $\gamma<\min (\beta, 1 / 2)$, there is a finite random constant $c>0$ such that the inequality

$$
\left\|Y-Y_{J}^{W}\right\|_{C^{\gamma}(I)} \leq c 2^{-J \min (\beta-\gamma, \alpha+\beta-1 / 2-\gamma)}
$$

\footnotetext{
${ }^{1}$ Observe that, for every $j \in \mathbb{N}$ and $k \in\left\{-N_{1}, \ldots, 2^{j} v-N_{2}\right\}$, one has

$$
\operatorname{supp} \psi\left(2^{j} \bullet-k\right) \subseteq\left[2^{-j}\left(N_{1}+k\right), 2^{-j}\left(N_{2}+k\right)\right] \subseteq I
$$
}

and consequently (see (2.7)) that

$$
a_{j, k}:=a_{j, k}(v)=2^{j / 2} \int_{\mathbb{R}} \psi\left(2^{j} s-k\right) \sigma(s) \mathrm{d} s .
$$


holds almost surely, for each $J \in \mathbb{N}$. We recall that the processes $Y:=\{Y(t)\}_{t \in I}$ and $Y_{J}^{W}:=\left\{Y_{J}^{W}(t)\right\}_{t \in I}$ have respectively been introduced in (1.1) and in Definition 2.1. Also, we recall that the interval $I:=[0, v]$.

In order to derive Theorem 3.5 one needs several preliminary results. First we are going to state all of them and then we will give their proofs.

The following lemma shows that for deriving the theorem it is enough to prove it when $\gamma=0$. Let us mention that this lemma remains valid even if one drops the Wiener chaos condition $(\mathcal{W} C)$.

Lemma 3.6. Assume that $\alpha, \beta$ and $\gamma$ are as in the statement of Theorem 3.5. Also, assume that there is a finite random constant $c^{\prime}>0$ such that the inequality

$$
\left\|Y-Y_{J}^{W}\right\|_{I, \infty} \leq c^{\prime} 2^{-J \min (\beta, \alpha+\beta-1 / 2)}
$$

holds almost surely, for each $J \in \mathbb{N}$. Then, there exists a finite random constant $c>0$ for which (3.7) is satisfied almost surely, for all $J \in \mathbb{N}$.

Let us now explain the strategy that will be employed to get the crucial inequality (3.8). To this end, for each fixed $j \in \mathbb{N}$, one denotes by $Z_{j}:=\left\{Z_{j}(t)\right\}_{t \in I}$ the stochastic process defined, for all $t \in I$, as

$$
Z_{j}(t):=\sum_{k=1-N_{2}}^{2^{j} v-N_{1}} a_{j, k}(t) \lambda_{j, k}=\sum_{k=1-N_{2}}^{\left[2^{j} t\right]-N_{1}} a_{j, k}(t) \lambda_{j, k},
$$

where the last equality follows from (2.7) and (2.8). Observe that $Z_{j}$ "lives" in the Wiener chaos $\overline{\mathcal{P}}_{2 n}$ (see Remark $3.4(c)$ ), and has continuous paths since the $a_{j, k}(\bullet, \omega)$ 's are continuous functions on $I$. Also, observe that one knows from the first equality in (2.13) and from Corollary 2.4 that the paths of the stochastic process $Y-Y_{J}^{W}$ can be expressed, for any fixed $J \in \mathbb{N}$ and $\omega \in \Omega$ (the underlying probability space), as the series of functions

$$
Y(\bullet, \omega)-Y_{J}^{W}(\bullet, \omega)=\sum_{j=J}^{+\infty} Z_{j}(\bullet, \omega),
$$

which converges in the Hölder space $C^{\gamma}(I)$, for any $\gamma \in[0, \beta)$. Therefore, using the triangle inequality, one has

$$
\left\|Y(\bullet, \omega)-Y_{J}^{W}(\bullet, \omega)\right\|_{I, \infty} \leq \sum_{j=J}^{+\infty}\left\|Z_{j}(\bullet, \omega)\right\|_{I, \infty} .
$$

Then it turns out that in order to get the crucial inequality (3.8) it is enough to obtain the following lemma.

Lemma 3.7. Assume that $\alpha$ and $\beta$ are as in the statement of Theorem 3.5. Then, one has almost surely

$$
\sup _{j \in \mathbb{N}}\left\{2^{j \min (\beta, \alpha+\beta-1 / 2)}\left\|Z_{j}\right\|_{I, \infty}\right\}<+\infty .
$$

Next, let us point out that $\left\|Z_{j}\right\|_{I, \infty}:=\sup _{t \in I}\left|Z_{j}(t)\right|$ is the supremum of infinitely many random variables. Actually, it is more convenient to work with a supremum of finite number of them; this can be done thanks to the following lemma. 
Lemma 3.8. For each $j \in \mathbb{N}$, one sets

$$
\nu\left(Z_{j}\right):=\sup _{l \in\left\{0, \ldots, 2^{j} v\right\}}\left|Z_{j}\left(2^{-j} l\right)\right| .
$$

Then, for any fixed $\beta \in\left(0, \beta_{0}\right)$, one has almost surely

$$
\sup _{j \in \mathbb{N}}\left\{2^{j \beta}\left|\left\|Z_{j}\right\|_{I, \infty}-\nu\left(Z_{j}\right)\right|\right\}<+\infty .
$$

Proof of Lemma 3.7: In view of Lemma 3.8, it turns out that in order to get Lemma 3.7 it is enough to obtain the following lemma.

Lemma 3.9. Assume that $\alpha$ and $\beta$ are as in the statement of Theorem 3.5. Then, one has almost surely

$$
\sup _{j \in \mathbb{N}}\left\{2^{j \min (\beta, \alpha+\beta-1 / 2)} \nu\left(Z_{j}\right)\right\}<+\infty .
$$

Notice that if one shows that

$$
\sum_{j=1}^{+\infty} \mathbb{P}\left(2^{j \min (\beta, \alpha+\beta-1 / 2)} \nu\left(Z_{j}\right)>1\right)<+\infty,
$$

then the Borel-Cantelli lemma entails that (3.14) holds. Using the Markov inequality, one has, for every $j \in \mathbb{N}$,

$$
\mathbb{P}\left(2^{j \min (\beta, \alpha+\beta-1 / 2)} \nu\left(Z_{j}\right)>1\right) \leq 2^{j \min (\beta, \alpha+\beta-1 / 2)} \mathbb{E}\left(\nu\left(Z_{j}\right)\right) .
$$

In view of (3.12) and of the fact that, for every fixed $j \in \mathbb{N}$, the random variables $Z_{j}\left(2^{-j} l\right), l \in\left\{0, \ldots, 2^{j} v\right\}$, "live" in the Wiener chaos $\overline{\mathcal{P}}_{2 n}$, one can see that, to get an appropriate upper bound for the expectation $\mathbb{E}\left(\nu\left(Z_{j}\right)\right)$, it suffices to use the following result with $n$ replaced by $2 n$.

Lemma 3.10. Let the integer $n \geq 1$ be arbitrary and fixed. There exists a universal deterministic finite constant $c(n)>0$, depending only on $n$, such that, for every sequence $\left\{\chi_{l}\right\}_{l \in \mathbb{N}}$ of random variables belonging to the nth Wiener chaos $\overline{\mathcal{P}}_{n}$, the inequality

$$
\mathbb{E}\left(\sup _{1 \leq l \leq L}\left|\chi_{l}\right|\right) \leq c(n) \log ^{n / 2}(8+L) \sup _{1 \leq l \leq L}\left(\mathbb{E}\left[\left|\chi_{l}\right|^{2}\right]\right)^{1 / 2}
$$

holds, for all $L \in \mathbb{N}$. Observe that $\log$ denotes Napierian logarithm.

Proof of Lemma 3.10: For each integer $L \geq 1$, one sets

$$
M_{L}:=\sup _{1 \leq l \leq L}\left|\chi_{l}\right|, \mu_{L}:=\sup _{1 \leq l \leq L}\left\|\chi_{l}\right\|_{L^{2}(\Omega)} \text { and } \lambda_{L}:=\frac{2 \log ^{n / 2}(8+L)}{\min \left\{\left(2^{-1} c_{1}(n)\right)^{n / 2}, 1\right\}},
$$

where $c_{1}(n)>0$ denotes the constant $c_{3}(n)$ in (3.2). There is no restriction to assume that $\mu_{L}>0$. Using Tonelli theorem for nonnegative functions and the change of variable $x=y \mu_{L}$, one has

$$
\mathbb{E}\left(M_{L}\right)=\int_{0}^{+\infty} \mathbb{P}\left(M_{L}>x\right) \mathrm{d} x=\mu_{L} \int_{0}^{+\infty} \mathbb{P}\left(M_{L}>y \mu_{L}\right) \mathrm{d} y .
$$

Thus, one gets that

$$
\mathbb{E}\left(M_{L}\right) \leq \mu_{L} \lambda_{L}+\mu_{L} \int_{\lambda_{L}}^{+\infty} \mathbb{P}\left(M_{L}>y \mu_{L}\right) \mathrm{d} y .
$$


Moreover, it follows from the first two equalities in (3.18) that, for all $y \in\left[\lambda_{L},+\infty\right)$, one has

$$
\mathbb{P}\left(M_{L}>y \mu_{L}\right)=\mathbb{P}\left(\bigcup_{l=1}^{L}\left\{\left|\chi_{l}\right|>y \mu_{L}\right\}\right) \leq \sum_{l=1}^{L} \mathbb{P}\left(\left|\chi_{l}\right|>y\left\|\chi_{l}\right\|_{L^{2}(\Omega)}\right) .
$$

On the other hand, one knows from the third equality in (3.18) that $\lambda_{L} \geq 2$. Thus, (3.2) and the third equality in (3.18) imply that, for all $l \in\{1, \ldots, L\}$,

$$
\begin{aligned}
& \int_{\lambda_{L}}^{+\infty} \mathbb{P}\left(\left|\chi_{l}\right|>y\left\|\chi_{l}\right\|_{L^{2}(\Omega)}\right) \mathrm{d} y \leq \int_{\lambda_{L}}^{+\infty} \exp \left(-c_{1}(n) y^{\frac{2}{n}}\right) \mathrm{d} y \\
& \leq c_{2}(n) \int_{\lambda_{L}}^{+\infty} y^{\frac{2}{n}-1} \exp \left(-2^{-1} c_{1}(n) y^{\frac{2}{n}}\right) \mathrm{d} y \\
& =c_{3}(n) \exp \left(-2^{-1} c_{1}(n) \lambda_{L}^{2 / n}\right) \\
& \leq c_{3}(n)(8+L)^{-1},
\end{aligned}
$$

where the constants $c_{2}(n):=\sup _{y \in[0,+\infty)} y \exp \left(-2^{-1} c_{1}(n) y^{2 / n}\right)<+\infty$ and $c_{3}(n):=$ $n c_{2}(n) / c_{1}(n)$. Next, one can derive from (3.20) and (3.21) that, for each $L \in \mathbb{Z}_{+}$,

$$
\int_{\lambda_{L}}^{+\infty} \mathbb{P}\left(M_{L}>y \mu_{L}\right) \mathrm{d} y \leq c_{3}(n) .
$$

Finally, putting together (3.18), (3.19) and (3.22) leads to (3.17).

Proof of Lemma 3.9: It follows from (3.12) and from Lemma 3.10 that, for all $j \in \mathbb{N}$, one has

$$
\mathbb{E}\left(\nu\left(Z_{j}\right)\right) \leq c(2 n)\left(\log \left(9+2^{j} v\right)\right)^{n} \sup _{l \in\left\{0, \ldots, 2^{j} v\right\}}\left(\mathbb{E}\left(\left|Z_{j}\left(2^{-j} l\right)\right|^{2}\right)\right)^{1 / 2} .
$$

Then, in view of (3.23), (3.16) and the inequalities $\alpha<\alpha_{0}$ and $\beta<\beta_{0}$, it turns out that in order to get (3.15) it is enough to obtain the following lemma.

Lemma 3.11. One has

$$
\sup _{j \in \mathbb{N}} \sup _{l \in\left\{0, \ldots, 2^{j} v\right\}}\left\{2^{2 j \min \left(\beta_{0}, \alpha_{0}+\beta_{0}-1 / 2\right)} \mathbb{E}\left(\left|Z_{j}\left(2^{-j} l\right)\right|^{2}\right)\right\}<+\infty .
$$

We mention in passing that the condition $(\mathcal{W C})$ will play a crucial role in the proof of Lemma 3.11.

Having stated the preliminary results one needs for getting Theorem 3.5 and having explained the connections between these preliminary results, the proof of the theorem then becomes quite straightforward.

Proof of Theorem 3.5: The theorem is a straightforward consequence of Lemma 3.6, (3.10) and Lemma 3.7.

From now on, one focuses on the proofs of Lemmas 3.6, 3.8 and 3.11.

Proof of Lemma 3.6: In view of (1.2), it is enough to show that, for every fixed nonnegative real number $\gamma<\min (\beta, 1 / 2)$, there is a finite random constant $c>0$ 
such that, for all $t_{1}, t_{2} \in I$ with $t_{1}<t_{2}$, and for each $J \in \mathbb{N}$, one has

$$
\begin{aligned}
R_{J}\left(t_{2}, t_{1}\right) & :=\frac{\left|Y\left(t_{2}\right)-Y\left(t_{1}\right)-Y_{J}^{W}\left(t_{2}\right)+Y_{J}^{W}\left(t_{1}\right)\right|}{\left(t_{2}-t_{1}\right)^{\gamma}} \\
& \leq c 2^{-J \min (\beta-\gamma, \alpha+\beta-1 / 2-\gamma)} .
\end{aligned}
$$

Notice that, without any restriction, it can be further assumed that $t_{2}-t_{1}<1$; indeed, when $t_{2}-t_{1} \geq 1$, it easily follows from the equality in (3.25), the triangle inequality, (1.3) and (3.8) that the inequality in (3.25) holds, for any finite random constant $c \geq 2 c^{\prime}$ and for all $J \in \mathbb{N}$.

Let $J_{0}$ be the unique positive integer such that

$$
2^{-J_{0}} \leq t_{2}-t_{1}<2^{-\left(J_{0}-1\right)} .
$$

Observe that, in the case where $J \geq J_{0}$, using the equality in (3.25), (3.26), the triangle inequality, (1.3) and (3.8), one has

$$
R_{J}\left(t_{2}, t_{1}\right) \leq 2^{1+J \gamma}\left\|Y-Y_{J}^{W}\right\|_{I, \infty} \leq\left(2 c^{\prime}\right) 2^{-J \min (\beta-\gamma, \alpha+\beta-1 / 2-\gamma)},
$$

which proves that the inequality in (3.25) holds in this case, for any finite random constant $c \geq 2 c^{\prime}$. From now on, one assumes that $J<J_{0}$. Then, from (3.26) and (2.16), it turns out that $\mathbb{L}_{j, t_{1}, t_{2}}$ is the empty set, for all $j \in\left\{J, \ldots, J_{0}-1\right\}$. Therefore, in view of (2.17), one has, for every $j \in\left\{J, \ldots, J_{0}-1\right\}$,

$$
\partial \mathbb{L}_{j, t_{1}, t_{2}}=\left\{k \in\left\{1-N_{2}, \ldots, 2^{j} v-N_{1}\right\}:\left[\frac{k+N_{1}}{2^{j}}, \frac{k+N_{2}}{2^{j}}\right] \cap\left[t_{1}, t_{2}\right] \neq \emptyset\right\} .
$$

Next, one sets

$$
S_{J_{0}, J}\left(t_{2}, t_{1}\right):=\left|Y_{J_{0}}^{W}\left(t_{2}\right)-Y_{J_{0}}^{W}\left(t_{1}\right)-Y_{J}^{W}\left(t_{2}\right)+Y_{J}^{W}\left(t_{1}\right)\right| .
$$

It results from the equality in (3.25), the triangle inequality and (3.27) that

$$
\begin{aligned}
& R_{J}\left(t_{2}, t_{1}\right) \leq R_{J_{0}}\left(t_{2}, t_{1}\right)+\left(t_{2}-t_{1}\right)^{-\gamma} S_{J_{0}, J}\left(t_{2}, t_{1}\right) \\
& \leq\left(2 c^{\prime}\right) 2^{-J_{0} \min (\beta-\gamma, \alpha+\beta-1 / 2-\gamma)}+\left(t_{2}-t_{1}\right)^{-\gamma} S_{J_{0}, J}\left(t_{2}, t_{1}\right) \\
& \leq\left(2 c^{\prime}\right) 2^{-J \min (\beta-\gamma, \alpha+\beta-1 / 2-\gamma)}+\left(t_{2}-t_{1}\right)^{-\gamma} S_{J_{0}, J}\left(t_{2}, t_{1}\right) .
\end{aligned}
$$

Let us now provide an appropriate upper bound for the random quantity $S_{J_{0}, J}\left(t_{2}, t_{1}\right)$. Using (3.29), the first equality in (2.13), (2.7), (2.8), (2.14), (3.28), the triangle inequality, $(2.20),(1.3),(2.40)$ and (3.26), one gets that

$$
\begin{aligned}
S_{J_{0}, J}\left(t_{2}, t_{1}\right) & \leq \sum_{j=J}^{J_{0}-1} 2^{j} \sum_{k \in \partial \mathbb{L}_{j, t_{1}, t_{2}}}\left|\int_{2^{-j}\left(k+N_{1}\right)}^{2^{-j}\left(k+N_{2}\right)} \sigma(s) \psi\left(2^{j} s-k\right) \mathbf{1}_{\left[t_{1}, t_{2}\right]}(s) \mathrm{d} s\right| \\
& \leq c_{1}\|\sigma\|_{I, \infty}\|\psi\|_{\left[N_{1}, N_{2}\right], \infty}\left(t_{2}-t_{1}\right) \sum_{j=J}^{J_{0}-1} 2^{j(1-\beta)} \\
& \leq c_{1}\|\sigma\|_{I, \infty}\|\psi\|_{\left[N_{1}, N_{2}\right], \infty}\left(t_{2}-t_{1}\right)^{\gamma} \sum_{j=J}^{J_{0}-1} 2^{-j(\beta-\gamma)} \mathrm{d} X(s) \mid \\
& \leq c_{2}\left(t_{2}-t_{1}\right)^{\gamma} 2^{-J(\beta-\gamma)},
\end{aligned}
$$


where

$$
c_{2}:=c_{1}\|\sigma\|_{I, \infty}\|\psi\|_{\left[N_{1}, N_{2}\right], \infty} \sum_{n=0}^{+\infty} 2^{-n(\beta-\gamma)}<+\infty,
$$

and $c_{1}$ is a finite random constant not depending on $J, t_{1}$ and $t_{2}$.

Finally, setting $c:=2 c^{\prime}+c_{2}$, one can derive from (3.27), (3.30) and (3.31) that the inequality in (3.25) is satisfied.

Proof of Lemma 3.8: Let us fix an arbitrary $j \in \mathbb{N}$. The fact that the paths of the process $\left\{Z_{j}(t)\right\}_{t \in I}$ are continuous functions on the compact interval $I$ implies that there exists a random point $t_{0} \in I$ such that

$$
\left\|Z_{j}\right\|_{I, \infty}:=\sup _{t \in I}\left|Z_{j}(t)\right|=\left|Z_{j}\left(t_{0}\right)\right| .
$$

Let us set $l_{0}:=\left[2^{j} t_{0}\right]$. Using (3.32), (3.12), the inequality $\nu\left(Z_{j}\right) \leq\left\|Z_{j}\right\|_{I, \infty}$ and the triangle inequality, one has

$$
||\left|Z_{j} \|_{I, \infty}-\nu\left(Z_{j}\right)\right| \leq|| Z_{j}\left(t_{0}\right)|-| Z_{j}\left(2^{-j} l_{0}\right)|| \leq\left|Z_{j}\left(t_{0}\right)-Z_{j}\left(2^{-j} l_{0}\right)\right| .
$$

Moreover, it follows from (3.9), (2.14) and (2.20) that

$$
\begin{aligned}
\left|Z_{j}\left(t_{0}\right)-Z_{j}\left(2^{-j} l_{0}\right)\right| & \leq \sum_{k=1-N_{2}}^{l_{0}-N_{1}}\left|a_{j, k}\left(t_{0}\right)-a_{j, k}\left(2^{-j} l_{0}\right)\right|\left|\lambda_{j, k}\right| \\
& \leq c_{1} 2^{-(\beta-1 / 2) j} \sum_{k=1-N_{2}}^{l_{0}-N_{1}}\left|a_{j, k}\left(t_{0}\right)-a_{j, k}\left(2^{-j} l_{0}\right)\right| \\
& =c_{1} 2^{-(\beta-1 / 2) j} \sum_{k=l_{0}-N_{2}+1}^{l_{0}-N_{1}}\left|a_{j, k}\left(t_{0}\right)-a_{j, k}\left(2^{-j} l_{0}\right)\right|
\end{aligned}
$$

where $c_{1}$ denotes the finite random constant $c_{1}^{\prime}$ in (2.20), and where the equality results from the fact that $a_{j, k}\left(t_{0}\right)=a_{j, k}\left(2^{-j} l_{0}\right)$, when $k \leq l_{0}-N_{2}$ (see (2.7) and (2.8)). Next observe that, using (2.7), (1.3) and the equality $l_{0}:=\left[2^{j} t_{0}\right]$, one has, for each $k \in\left\{l_{0}-N_{2}+1, \ldots, l_{0}-N_{1}\right\}$, that

$$
\begin{aligned}
\left|a_{j, k}\left(t_{0}\right)-a_{j, k}\left(2^{-j} l_{0}\right)\right| & =2^{j / 2}\left|\int_{2^{-j} l_{0}}^{t_{0}} \sigma(s) \psi\left(2^{j} s-k\right) \mathrm{d} s\right| \\
& \leq 2^{j / 2}\left(t_{0}-2^{-j} l_{0}\right)\|\sigma\|_{I, \infty}\|\psi\|_{\left[N_{1}, N_{2}\right], \infty} \\
& \leq 2^{-j / 2}\|\sigma\|_{I, \infty}\|\psi\|_{\left[N_{1}, N_{2}\right], \infty} .
\end{aligned}
$$

Thus, letting $c_{2}$ be the finite random constant, not depending on $j$, defined as $c_{2}:=c_{1}\left(N_{2}-N_{1}\right)\|\sigma\|_{I, \infty}\|\psi\|_{\left[N_{1}, N_{2}\right], \infty}$, one can derive from (3.33) and (3.34) that

$$
\left|\left\|Z_{j}\right\|_{I, \infty}-\nu\left(Z_{j}\right)\right| \leq c_{2} 2^{-j \beta}
$$

which proves that (3.13) holds.

In order to derive Lemma 3.11, one needs the following lemma.

Lemma 3.12. Assume that $\left(\mathcal{C}_{1}\right)$ in Definition 3.3 holds. Then, there is a finite deterministic constant $c>0$ such that, for every $t \in I$ and $j \in \mathbb{N}$, one has

$$
\mathbb{E}\left[\left|\lambda_{j, k}\right|^{2}\right] \leq c 2^{-j\left(2 \beta_{0}-1\right)}, \quad \text { for all } k \in\left\{1-N_{2}, \ldots,\left[2^{j} v\right]-N_{1}\right\}
$$




$$
\mathbb{E}\left[\left|a_{j, k}(t)\right|^{2}\right] \leq c 2^{-j\left(2 \alpha_{0}+1\right)}, \quad \text { for all } k \in \mathbb{L}_{j, 0, t},
$$

and

$$
\mathbb{E}\left[\left|a_{j, k}(t)\right|^{2}\right] \leq c 2^{-j}, \quad \text { for all } k \in \partial \mathbb{L}_{j, 0, t},
$$

where the sets $\mathbb{L}_{j, 0, t}$ and $\partial \mathbb{L}_{j, 0, t}$ are defined through (2.16) and (2.17), with $J=j$, $t_{1}=0$ and $t_{2}=t$.

Proof of Lemma 3.12: It is clear that (3.35) holds when $\psi$ satisfies the hypothesis $\left(\mathcal{H}_{1}\right)$ (see the beginning of Section 2). The proof of (3.35) under the hypothesis $\left(\mathcal{H}_{2}\right)$ is long and technical. For the sake of simplicity we only gives it in the case where $\psi$ is continuously differentiable. Denoting by $\psi^{\prime}$ the derivative of $\psi$, in view of (2.14), one has almost surely that

$$
\lambda_{j, k}=-2^{3 j / 2} \int_{2^{-j}\left(k+N_{1}\right)}^{2^{-j}\left(k+N_{2}\right)} X(s) \psi^{\prime}\left(2^{j} s-k\right) \mathrm{d} s .
$$

Then, the change of variable $u=2^{j} s-k$ and the equality $\int_{N_{1}}^{N_{2}} \psi^{\prime}(u) \mathrm{d} u=0$ imply almost surely that

$$
\begin{aligned}
\lambda_{j, k} & =-2^{j / 2} \int_{N_{1}}^{N_{2}} X\left(2^{-j}(u+k)\right) \psi^{\prime}(u) \mathrm{d} u \\
& =-2^{j / 2} \int_{N_{1}}^{N_{2}}\left(X\left(2^{-j}(u+k)\right)-X\left(2^{-j} k\right)\right) \psi^{\prime}(u) \mathrm{d} u .
\end{aligned}
$$

Thus, setting $c_{1}:=\int_{N_{1}}^{N_{2}}\left|\psi^{\prime}(u)\right|^{2} \mathrm{~d} u$ and applying Cauchy-Schwarz inequality as well as Tonelli theorem for nonnegative functions, one obtains that

$$
\mathbb{E}\left[\left|\lambda_{j, k}\right|^{2}\right] \leq c_{1} 2^{j} \int_{N_{1}}^{N_{2}} \mathbb{E}\left[\left|X\left(2^{-j}(u+k)\right)-X\left(2^{-j} k\right)\right|^{2}\right] \mathrm{d} u .
$$

Then (3.35) follows from (3.4).

We skip the proof of (3.36) since it is rather similar to that of (3.35).

Let us now turn to the proof of (3.37). Assume that $k \in \partial \mathbb{L}_{j, 0, t}$. Using (2.7), the change of variable $u=2^{j} s-k$, and the second inclusion in (2.8), one has

$$
\left|a_{j, k}(t)\right| \leq 2^{j / 2} \int_{-\infty}^{+\infty}|\sigma(s)|\left|\psi\left(2^{j} s-k\right)\right| \mathrm{d} s=2^{-j / 2} \int_{N_{1}}^{N_{2}}\left|\sigma\left(2^{-j}(u+k)\right)\right||\psi(u)| \mathrm{d} u .
$$

Thus, it follows from Cauchy-Schwarz inequality as well as Tonelli theorem for nonnegative functions that

$$
\mathbb{E}\left[\left|a_{j, k}(t)\right|^{2}\right] \leq c_{2} 2^{-j} \int_{N_{1}}^{N_{2}} \mathbb{E}\left[\left|\sigma\left(2^{-j}(u+k)\right)\right|^{2}\right] \mathrm{d} u,
$$

where $c_{2}:=\int_{N_{1}}^{N_{2}}|\psi(u)|^{2} \mathrm{~d} u$. On the other hand, observe that (3.3) entails that $x \mapsto \mathbb{E}\left[|\sigma(x)|^{2}\right]$ is a continuous function on $\mathbb{R}$, and consequently a bounded function on each compact interval. Therefore, (3.37) results from (3.38) and (2.17).

Proof of Lemma 3.11: Assume that $j \in \mathbb{N}$ and $l \in\left\{0, \ldots, 2^{j} v\right\}$ are arbitrary and fixed. Using (3.9), the triangle inequality and the inequality $(x+y)^{2} \leq 2 x^{2}+2 y^{2}$, 
for all $x, y \in \mathbb{R}_{+}$, one obtains that

$$
\begin{aligned}
& \mathbb{E}\left[\left|Z_{j}\left(2^{-j} l\right)\right|^{2}\right]=\mathbb{E}\left[\left|\sum_{k \in \mathbb{L}_{j, l}} a_{j, k}\left(2^{-j} l\right) \lambda_{j, k}+\sum_{k \in \partial \mathbb{L}_{j, l}} a_{j, k}\left(2^{-j} l\right) \lambda_{j, k}\right|^{2}\right] \\
& \leq 2 \mathbb{E}\left[\left|\sum_{k \in \mathbb{L}_{j, l}} a_{j, k}\left(2^{-j} l\right) \lambda_{j, k}\right|^{2}\right]+2 \mathbb{E}\left[\left|\sum_{k \in \partial \mathbb{L}_{j, l}} a_{j, k}\left(2^{-j} l\right) \lambda_{j, k}\right|^{2}\right]
\end{aligned}
$$

where $\mathbb{L}_{j, l}$ and $\partial \mathbb{L}_{j, l}$ denote the two sets of indices defined as $\mathbb{L}_{j, l}:=\mathbb{L}_{j, 0,2^{-j} l}$ and $\partial \mathbb{L}_{j, l}:=\partial \mathbb{L}_{j, 0,2^{-j} l}$ (see (2.16) and (2.17)). Next, observe that, when $k \in \mathbb{L}_{j, l}$, one has $a_{j, k}\left(2^{-j} l\right)=a_{j, k}:=a_{j, k}(v)$. Thus, using the inclusion $\mathbb{L}_{j, l} \subseteq\left\{-N_{1}, \ldots, 2^{j} v-\right.$ $\left.N_{2}\right\}$ and (3.6), one gets that

$$
\begin{aligned}
& \mathbb{E}\left[\left|\sum_{k \in \mathbb{L}_{j, l}} a_{j, k}\left(2^{-j} l\right) \lambda_{j, k}\right|^{2}\right] \\
& =\sum_{k_{1} \in \mathbb{L}_{j, l}} \sum_{k_{2} \in \mathbb{L}_{j, l}} \mathbb{E}\left[a_{j, k_{1}} \lambda_{j, k_{1}} a_{j, k_{2}} \lambda_{j, k_{2}}\right] \leq \sum_{k_{1} \in \mathbb{L}_{j, l}} \sum_{k_{2} \in \mathbb{L}_{j, l}}\left|\mathbb{E}\left[a_{j, k_{1}} \lambda_{j, k_{1}} a_{j, k_{2}} \lambda_{j, k_{2}}\right]\right| \\
& \leq \sum_{k_{1}=-N_{1}}^{2^{j} v-N_{2}} \sum_{2_{2}=-N_{1}}^{2_{v}}\left|\mathbb{E}\left[a_{j, k_{1}} \lambda_{j, k_{1}} a_{j, k_{2}} \lambda_{j, k_{2}}\right]\right| \leq c_{1} 2^{-2 j\left(\alpha_{0}+\beta_{0}-1 / 2\right)}
\end{aligned}
$$

where $c_{1}$ is a finite constant not depending on $j$ and $l$.

Let us now provide an appropriate upper bound for the second term in the righthand side of (3.39). First, observe that, as done in (2.40), it can be shown that there is a finite deterministic constant $c_{2}>0$, not depending on $j$ and $l$, such that one has

$$
\operatorname{card}\left(\partial \mathbb{L}_{j, l}\right) \leq c_{2}
$$

Also, observe that it follows from the Cauchy-Schwarz inequality and the second inequality in (3.1) that for all $j \in \mathbb{N}, k_{1}, k_{2} \in\left\{1-N_{2}, \ldots, 2^{j} v-N_{1}\right\}$, and $l \in$ $\left\{0, \ldots, 2^{j} v\right\}$, one has

$$
\begin{aligned}
& \left|\mathbb{E}\left[a_{j, k_{1}}\left(2^{-j} l\right) \lambda_{j, k_{1}} a_{j, k_{2}}\left(2^{-j} l\right) \lambda_{j, k_{2}}\right]\right| \\
& \leq\left(\mathbb{E}\left[\left|a_{j, k_{1}}\left(2^{-j} l\right)\right|^{4}\right] \mathbb{E}\left[\left|\lambda_{j, k_{1}}\right|^{4}\right] \mathbb{E}\left[\left|a_{j, k_{2}}\left(2^{-j} l\right)\right|^{4}\right] \mathbb{E}\left[\left|\lambda_{j, k_{2}}\right|^{4}\right]\right)^{\frac{1}{4}} \\
& \leq c_{3}\left(\mathbb{E}\left[\left|a_{j, k_{1}}\left(2^{-j} l\right)\right|^{2}\right] \mathbb{E}\left[\left|\lambda_{j, k_{1}}\right|^{2}\right] \mathbb{E}\left[\left|a_{j, k_{2}}\left(2^{-j} l\right)\right|^{2}\right] \mathbb{E}\left[\left|\lambda_{j, k_{2}}\right|^{2}\right]\right)^{\frac{1}{2}},
\end{aligned}
$$

where $c_{3}$ is a finite constant not depending on $j, k_{1}, k_{2}$ and $l$. Thus, when $k_{1}, k_{2} \in$ $\partial \mathbb{L}_{j, l}$, combining (3.42) with (3.35) and (3.37) one obtains

$$
\begin{aligned}
\left|\mathbb{E}\left[a_{j, k_{1}}\left(2^{-j} l\right) \lambda_{j, k_{1}} a_{j, k_{2}}\left(2^{-j} l\right) \lambda_{j, k_{2}}\right]\right| & \leq c_{3}\left(c 2^{-j} c 2^{-j\left(2 \beta_{0}-1\right)} c 2^{-j} c 2^{-j\left(2 \beta_{0}-1\right)}\right)^{1 / 2} \\
& =c_{4} 2^{-2 j \beta_{0}}
\end{aligned}
$$


where $c_{4}=c_{3} c^{2}$ is a finite constant not depending on $j, k_{1}, k_{2}$ and $l$, with $c$ being the constant given in Lemma 3.12. Next, it follows from (3.41) and (3.43) that

$$
\begin{aligned}
& \mathbb{E}\left[\left|\sum_{k \in \partial \mathbb{L}_{j, l}} a_{j, k}\left(2^{-j} l\right) \lambda_{j, k}\right|^{2}\right] \\
& \leq \sum_{k_{1} \in \partial \mathbb{L}_{j, l}} \sum_{k_{2} \in \partial \mathbb{L}_{j, l}}\left|\mathbb{E}\left[a_{j, k_{1}}\left(2^{-j} l\right) \lambda_{j, k_{1}} a_{j, k_{2}}\left(2^{-j} l\right) \lambda_{j, k_{2}}\right]\right| \\
& \leq c_{5} 2^{-2 j \beta_{0}}
\end{aligned}
$$

where $c_{5}=c_{2}^{2} c_{4}$ is a finite constant not depending on $j$ and $l$.

Finally, putting together (3.39), (3.40) and (3.44) one gets (3.24).

\section{Examples of processes satisfying the condition $(\mathcal{W} C)$}

This section involves constructing wide classes of examples of real-valued stochastic processes $\sigma$ and $X$ satisfying the Wiener chaos condition $(\mathcal{W} C)$ described in Definition 3.3. For the sake of convenience one assumes that these two processes are independent, centred and given by multiple Itô-Wiener integrals (see for example Janson, 1997; Nualart, 1995) in the frequency domain. More precisely, one denotes by $\left\{\mathbb{W}_{\sigma}(u)\right\}_{u \in \mathbb{R}}$ and $\left\{\mathbb{W}_{X}(u)\right\}_{u \in \mathbb{R}}$ two independent real-valued Brownian motions defined on the probability space $(\Omega, \mathcal{F}, \mathbb{P})$. Then, for $\mu=\sigma$ or $\mu=X$, one lets $\widehat{\mathbb{W}}_{\mu}$ be the complex-valued Gaussian random measure, defined as the "Fourier transform" of $\mathbb{W}_{\mu}$, that is:

$$
\sqrt{2 \pi} \widehat{\mathbb{W}}_{\mu}(A):=\int_{\mathbb{R}}\left(\int_{A} \cos (u \eta) \mathrm{d} \eta\right) \mathrm{d} \mathbb{W}_{\mu}(u)+i \int_{\mathbb{R}}\left(\int_{A} \sin (u \eta) \mathrm{d} \eta\right) \mathrm{d} \mathbb{W}_{\mu}(u),
$$

for each Borel set $A$ with a finite Lebesgue measure; notice that $\int_{\mathbb{R}}(\cdot) d \mathbb{W}_{\mu}$ is the Wiener integral associated with the Brownian motion $\mathbb{W}_{\mu}$. Recall that $\{\mu(s)\}_{s \in \mathbb{R}}$ denotes either the process $\{\sigma(s)\}_{s \in \mathbb{R}}$ or the process $\{X(s)\}_{s \in \mathbb{R}}$. It is assumed to be, for some positive integer $N_{\mu}$ and for each $s \in \mathbb{R}$, of the following form:

$$
\mu(s)=\int_{\mathbb{R}^{N_{\mu}}}\left(e^{i s\left(\eta_{1}+\ldots+\eta_{N_{\mu}}\right)}-1\right) g_{\mu}\left(\eta_{1}, \ldots, \eta_{N_{\mu}}\right) \mathrm{d} \widehat{\mathbb{W}}_{\mu}\left(\eta_{1}\right) \ldots \mathrm{d} \widehat{\mathbb{W}}_{\mu}\left(\eta_{N_{\mu}}\right),
$$

where $g_{\mu}$ is a Borel function from $\mathbb{R}^{N_{\mu}}$ into $\mathbb{C}$ (the set of the complex numbers), which satisfies the following three properties ${ }^{2}$.

(i) One has

$$
\int_{\mathbb{R}^{N_{\mu}}} \min \left(1,\left(\eta_{1}+\ldots+\eta_{N_{\mu}}\right)^{2}\right)\left|g\left(\eta_{1}, \ldots, \eta_{N_{\mu}}\right)\right|^{2} \mathrm{~d} \eta_{1} \ldots \mathrm{d} \eta_{N_{\mu}}<+\infty .
$$

(ii) The function $g_{\mu}$ is symmetric in its variables $\eta_{1}, \ldots, \eta_{N_{\mu}}$, i.e. the quantity $g_{\mu}\left(\eta_{1}, \ldots, \eta_{N_{\mu}}\right)$ remains the same when $\eta_{1}, \ldots, \eta_{N_{\mu}}$ are interchanged.

(iii) Denoting by $\overline{g_{\mu}\left(\eta_{1}, \ldots, \eta_{N_{\mu}}\right)}$ the complex-conjugate of $g_{\mu}\left(\eta_{1}, \ldots, \eta_{N_{\mu}}\right)$, one has, for almost all $\left(\eta_{1}, \ldots, \eta_{N_{\mu}}\right)$,

$$
\overline{g_{\mu}\left(\eta_{1}, \ldots, \eta_{N_{\mu}}\right)}=g_{\mu}\left(-\eta_{1}, \ldots,-\eta_{N_{\mu}}\right) .
$$

\footnotetext{
${ }^{2}$ Notice that these properties guarantee the existence of the multiple Itô-Wiener integral in (4.1) and the fact that it is real-valued.
} 
Remark 4.1. It is worth mentioning that the well-known Gaussian fractional Brownian motion of an arbitrary Hurst parameter $H \in(0,1)$ belongs to the class of processes defined through (4.1): in its case, one has $N_{\mu}=1$ and $g_{\mu}(\eta)=c|\eta|^{-H-1 / 2}$, for almost all $\eta \in \mathbb{R}$, where $c$ is an arbitrary nonvanishing constant. Also we mention that the non-Gaussian Rosenblatt process of an arbitrary parameter $d \in(1 / 4,1 / 2)$ belongs to this same class of processes: in its case one has $N_{\mu}=2$ and $g_{\mu}\left(\eta_{1}, \eta_{2}\right)=$ $-i\left(\eta_{1}+\eta_{2}\right)^{-1}\left|\eta_{1} \eta_{2}\right|^{-d}$, for almost all $\left(\eta_{1}, \eta_{2}\right) \in \mathbb{R}^{2}$.

Let us now observe that it follows from the "isometry property" of the multiple Itô-Wiener integral in (4.1) and from the change of variable $\xi=\eta_{1}+\eta_{2}+\ldots+\eta_{N_{\mu}}$, that, for all $\left(s_{1}, s_{2}\right) \in \mathbb{R}^{2}$, one has

$$
\begin{aligned}
& \mathbb{E}\left[\mu\left(s_{1}\right) \mu\left(s_{2}\right)\right] \\
& =\left(N_{\mu}\right) ! \int_{\mathbb{R}^{N_{\mu}}}\left(e^{i s_{1}\left(\eta_{1}+\eta_{2}+\ldots+\eta_{N_{\mu}}\right)}-1\right)\left(e^{-i s_{2}\left(\eta_{1}+\eta_{2}+\ldots+\eta_{N_{\mu}}\right)}-1\right) \\
& \quad \times\left|g_{\mu}\left(\eta_{1}, \eta_{2}, \ldots, \eta_{N_{\mu}}\right)\right|^{2} \mathrm{~d} \eta_{1} \mathrm{~d} \eta_{2} \ldots \mathrm{d} \eta_{N_{\mu}} \\
& =\left(N_{\mu}\right) ! \int_{\mathbb{R}^{N_{\mu}}}\left(e^{i s_{1} \xi}-1\right)\left(e^{-i s_{2} \xi}-1\right) \\
& \quad \times\left|g_{\mu}\left(\xi-\eta_{2}-\ldots-\eta_{N_{\mu}}, \eta_{2}, \ldots, \eta_{N_{\mu}}\right)\right|^{2} \mathrm{~d} \xi \mathrm{d} \eta_{2} \ldots \mathrm{d} \eta_{N_{\mu}} .
\end{aligned}
$$

Next, one denotes by $f_{\mu}$ the even ${ }^{3}$ and positive Borel function defined, for each $\xi \in \mathbb{R}$, as

$$
f_{\mu}(\xi):=\left(N_{\mu}\right) ! \int_{\mathbb{R}^{N_{\mu}-1}}\left|g_{\mu}\left(\xi-\eta_{2}-\ldots-\eta_{N_{\mu}}, \eta_{2}, \ldots, \eta_{N_{\mu}}\right)\right|^{2} \mathrm{~d} \eta_{2} \ldots \mathrm{d} \eta_{N_{\mu}},
$$

with the convention that $f_{\mu}(\xi):=\left|g_{\mu}(\xi)\right|^{2}$, when $N_{\mu}=1$. On one hand, one can easily derive from (4.2) and (4.4) that

$$
\int_{\mathbb{R}} \min \left(1, \xi^{2}\right) f_{\mu}(\xi) d \xi<+\infty
$$

On the other hand, using (4.3), (4.4) and Fubini theorem one obtains, for all $\left(s_{1}, s_{2}\right) \in \mathbb{R}^{2}$, that

$$
\mathbb{E}\left[\mu\left(s_{1}\right) \mu\left(s_{2}\right)\right]=\int_{-\infty}^{+\infty}\left(e^{i s_{1} \xi}-1\right)\left(e^{-i s_{2} \xi}-1\right) f_{\mu}(\xi) \mathrm{d} \xi .
$$

In other words, one has

$$
\mathbb{E}\left[\left|\mu\left(s_{1}\right)-\mu\left(s_{2}\right)\right|^{2}\right]=\mathbb{E}\left[\left|\mu\left(\left|s_{1}-s_{2}\right|\right)\right|^{2}\right]=4 \int_{-\infty}^{+\infty} \sin ^{2}\left(\frac{\left|s_{1}-s_{2}\right| \xi}{2}\right) f_{\mu}(\xi) \mathrm{d} \xi
$$

Remark 4.2. Assume that the process $\{\mu(s)\}_{s \in \mathbb{R}}$ is self-similar of order $\gamma_{0} \in(0,1)$, that is the processes $\{\mu(a s)\}_{s \in \mathbb{R}}$ and $\left\{a^{\gamma_{0}} \mu(s)\right\}_{s \in \mathbb{R}}$ have the same finite-dimensional distributions, for any fixed positive real number $a$. Then, the corresponding function $f_{\mu}$ is more precisely denoted by $f_{\mu, S S\left(\gamma_{0}\right)}$ and satisfies, for almost all $\xi \in \mathbb{R}$, $f_{\mu, S S\left(\gamma_{0}\right)}(\xi)=c|\xi|^{-2 \gamma_{0}-1}$, where $c$ is some positive constant. We recall in passing that the Gaussian fractional Brownian motion of Hurst parameter $H \in(0,1)$ is

\footnotetext{
${ }^{3}$ This means that $f_{\mu}(\xi)=f_{\mu}(-\xi)$, for almost all $\xi \in \mathbb{R}$. Notice that the fact that $f_{\mu}$ is an even function implies that the integral in (4.6) is real-valued.
} 
self-similar of order $\gamma_{0}=H$. Also, we recall that non-Gaussian Rosenblatt process of parameter $d \in(1 / 4,1 / 2)$ is self-similar of order $\gamma_{0}=2 d$.

A simple sufficient condition on $f_{\sigma}$ and $f_{X}$ for the processes $\sigma$ and $X$ to satisfy $\left(\mathcal{C}_{1}\right)$ in Definition 3.3 is provided by the following remark.

Remark 4.3. Let $\alpha_{0}, \beta_{0} \in(0,1)$ be as in Definition 3.3. A sufficient condition for the process $\sigma$ to satisfy (3.3) is the following: there exist two positive finite deterministic constants $c$ and $\xi_{0}$, such that the inequality

$$
f_{\sigma}(\xi) \leq c|\xi|^{-2 \alpha_{0}-1}
$$

holds for almost all real number $\xi$ satisfying $|\xi| \geq \xi_{0}$. Notice that in order to get a sufficient condition for the process $X$ to satisfy (3.4), one simply has to replace in (4.8) $\sigma$ by $X$ and $\alpha_{0}$ by $\beta_{0}$. Also notice that, in view of Remark 4.2, the inequality (4.8) holds as soon as the process $\sigma$ is self-similar of order $\gamma_{0} \geq \alpha_{0}$.

Proof of Remark 4.3: Let $s_{1}$ and $s_{2}$ be two arbitrary and distinct ${ }^{4}$ real numbers belonging to some fixed compact interval $\mathcal{K}$. It can easily be derived from (4.7) with $\mu=\sigma$, from (4.8) and from the classical inequality $|\sin (x)| \leq|x|$, for all $x \in \mathbb{R}$, that

$$
\begin{aligned}
& \mathbb{E}\left[\left|\sigma\left(s_{1}\right)-\sigma\left(s_{2}\right)\right|^{2}\right] \\
& \leq\left(s_{1}-s_{2}\right)^{2} \int_{-\xi_{0}}^{\xi_{0}} \xi^{2} f_{\sigma}(\xi) \mathrm{d} \xi+4 c \int_{-\infty}^{+\infty} \sin ^{2}\left(\frac{\left|s_{1}-s_{2}\right| \xi}{2}\right)|\xi|^{-2 \alpha_{0}-1} \mathrm{~d} \xi .
\end{aligned}
$$

Then, setting $\zeta=\left|s_{1}-s_{2}\right| \xi$ in the second integral, and using (4.5), the fact that $\alpha_{0} \in(0,1)$, and the inequalities $\left|s_{1}-s_{2}\right| \leq \operatorname{diam}(\mathcal{K})<+\infty$, one obtains (3.3).

From now on our goal is to provide sufficient conditions on $f_{\sigma}, f_{X}$ and the wavelet $\psi$ (see (3.5) and (2.14)) under which $\left(\mathcal{C}_{2}\right)$ in Definition 3.3 holds.

Remark 4.4. Let $\alpha_{0}, \beta_{0} \in(0,1)$ be as in Definition 3.3. Suppose there are a constant $c>0$ and two nonnegative integers $U_{0}$ and $V_{0}$ satisfying $U_{0}+V_{0}=2$, such that, for every $j \in \mathbb{N}$ and for all $k_{1}, k_{2} \in\left\{-N_{1}, \ldots, 2^{j} v-N_{2}\right\}$, one has

$$
\left|\mathbb{E}\left[a_{j, k_{1}} a_{j, k_{2}}\right]\right| \leq c 2^{-j\left(2 \alpha_{0}+1\right)}\left(1+\left|k_{1}-k_{2}\right|\right)^{-U_{0}}
$$

and

$$
\left|\mathbb{E}\left[\lambda_{j, k_{1}} \lambda_{j, k_{2}}\right]\right| \leq c 2^{-j\left(2 \beta_{0}-1\right)}\left(1+\left|k_{1}-k_{2}\right|\right)^{-V_{0}} .
$$

Then (3.6) is satisfied.

Proof of Remark 4.4: The fact that $\{\sigma(s)\}_{s \in I}$ and $\{X(s)\}_{s \in I}$ are independent implies that the $a_{j, k}$ 's and $\lambda_{j, k}$ 's are independent. This together with the inequalities (4.9), (4.10) and the fact that $U_{0}+V_{0}=2$ yields

$$
\begin{aligned}
& \left|\mathbb{E}\left[a_{j, k_{1}} \lambda_{j, k_{1}} a_{j, k_{2}} \lambda_{j, k_{2}}\right]\right|=\left|\mathbb{E}\left[a_{j, k_{1}} a_{j, k_{2}}\right]\right|\left|\mathbb{E}\left[\lambda_{j, k_{1}} \lambda_{j, k_{2}}\right]\right| \\
& \leq c^{2} 2^{-2 j\left(\alpha_{0}+\beta_{0}\right)}\left(1+\left|k_{1}-k_{2}\right|\right)^{-2},
\end{aligned}
$$

for all $k_{1}, k_{2} \in\left\{-N_{1}, \ldots, 2^{j} v-N_{2}\right\}$. Then (3.6) can be straightforwardly obtained by using (4.11).

\footnotetext{
${ }^{4}$ It is clear that (3.3) holds when $s_{1}=s_{2}$.
} 
The following four propositions are the four main results of the present section.

Proposition 4.5. Assume that the wavelet $\psi$ in (2.14) is the Haar function, that is $\psi:=\mathbf{1}_{[0,1 / 2)}-\mathbf{1}_{[1 / 2,1)}$. Then (4.10) holds as soon as $f_{X}$ is $V_{0}$ times continuously differentiable on $\mathbb{R} \backslash\{0\}$ and satisfies the following condition $\left(\mathcal{D}_{1, X}\right)$, in which $f_{X}^{(n)}$ denotes the derivative of $f_{X}$ of order $n$, with the convention that $f_{X}^{(0)}:=f_{X}$.

$\left(\mathcal{D}_{1, X}\right)$ There exist two finite deterministic constants $\beta_{0}^{\prime} \in\left[\beta_{0}, 1\right)$ and $c>0$ such that, for all $n \in\left\{0, \ldots, V_{0}\right\}$ and $\xi \in \mathbb{R} \backslash\{0\}$, one has

$$
\left|f_{X}^{(n)}(\xi)\right| \leq c \max \left(|\xi|^{-2 \beta_{0}-n-1},|\xi|^{-2 \beta_{0}^{\prime}-n-1}\right) .
$$

Proposition 4.6. Let $M \in \mathbb{N}$ be arbitrary and fixed. Assume that the wavelet $\psi$ in (2.14) is continuously differentiable on the real line and has at least $M$ vanishing moments, that is

$$
\int_{-\infty}^{+\infty} s^{m} \psi(s) \mathrm{d} s=0, \quad \text { for all } m \in\{0, \ldots, M-1\} .
$$

Then (4.10) holds as soon as $f_{X}$ is $V_{0}$ times continuously differentiable on $\mathbb{R} \backslash\{0\}$ and satisfies the following condition $\left(\mathcal{D}_{M, X}\right)$, which is weaker ${ }^{5}$ than $\left(\mathcal{D}_{1, X}\right)$.

$\left(\mathcal{D}_{M, X}\right)$ There exist two finite deterministic constants $\beta_{0}^{\prime} \in\left[\beta_{0}, 1\right)$ and $c>0$ such that, for all $n \in\left\{0, \ldots, V_{0}\right\}$ and $\xi \in \mathbb{R} \backslash\{0\}$, one has

$$
\left|f_{X}^{(n)}(\xi)\right| \leq c \max \left(|\xi|^{-2 \beta_{0}-n-1},|\xi|^{-2 \beta_{0}^{\prime}-n M-1}\right) .
$$

Proposition 4.7. Assume that the wavelet $\psi$ in (3.5) is the Haar function. Also assume that the integer $U_{0}$ in (4.9) belongs to the set $\{0,1\}$. Then (4.9) holds as soon as $f_{\sigma}$ is $U_{0}$ times continuously differentiable on $\mathbb{R} \backslash\{0\}$ and satisfies the following condition $\left(\mathcal{D}_{1, \sigma}\right)$, in which $f_{\sigma}^{(n)}$ denotes the derivative of $f_{\sigma}$ of order $n$, with the convention that $f_{\sigma}^{(0)}:=f_{\sigma}$.

$\left(\mathcal{D}_{1, \sigma}\right)$ There exist two finite deterministic constants $\alpha_{0}^{\prime} \in\left[\alpha_{0}, 1\right)$ and $c>0$ such that, for all $n \in\left\{0, \ldots, U_{0}\right\}$ and $\xi \in \mathbb{R} \backslash\{0\}$, one has

$$
\left|f_{\sigma}^{(n)}(\xi)\right| \leq c \max \left(|\xi|^{-2 \alpha_{0}-n-1},|\xi|^{-2 \alpha_{0}^{\prime}-n-1}\right) .
$$

Proposition 4.8. Let $M \in \mathbb{N}$ be arbitrary and fixed. Assume that the wavelet $\psi$ in (3.5) is continuously differentiable on the real line and has at least $M+1$ vanishing moments. Then (4.9) holds as soon as $f_{\sigma}$ is $U_{0}$ times continuously differentiable on $\mathbb{R} \backslash\{0\}$ and satisfies the following condition $\left(\mathcal{D}_{M, \sigma}\right)$, which is weaker ${ }^{6}$ than $\left(\mathcal{D}_{1, \sigma}\right)$.

$\left(\mathcal{D}_{M, \sigma}\right)$ There exist two finite deterministic constants $\alpha_{0}^{\prime} \in\left[\alpha_{0}, 1\right)$ and $c>0$ such that, for all $n \in\left\{0, \ldots, U_{0}\right\}$ and $\xi \in \mathbb{R} \backslash\{0\}$, one has

$$
\left|f_{\sigma}^{(n)}(\xi)\right| \leq c \max \left(|\xi|^{-2 \alpha_{0}-n-1},|\xi|^{-2 \alpha_{0}^{\prime}-n M-1}\right) .
$$

Remark 4.9. For $\mu=\sigma$ or $\mu=X$, it is clear that $\left(\mathcal{D}_{1, \mu}\right)$ holds when $\mu$ is self-similar of order $\gamma_{0}$ (see Remark 4.2).

A major motivation for weakening the condition $\left(\mathcal{D}_{1, \mu}\right)$ to the condition $\left(\mathcal{D}_{M, \mu}\right)$ is the following: the behavior of $f_{\mu}$ in the neighborhood of 0 can then be much

\footnotetext{
${ }^{5}$ More generally, $\left(\mathcal{D}_{M, X}\right)$ is weaker than $\left(\mathcal{D}_{M^{\prime}, X}\right)$, for any $M^{\prime}<M$.

${ }^{6}$ More generally, $\left(\mathcal{D}_{M, \sigma}\right)$ is weaker than $\left(\mathcal{D}_{M^{\prime}, \sigma}\right)$, for any $M^{\prime}<M$.
} 
more singular, namely $f_{\mu}$ can have infinitely many oscillations in the vicinity of 0 . This is for instance the case, when, for all $\xi \in \mathbb{R} \backslash\{0\}$, one has

$$
f_{\mu}(\xi)=|\xi|^{-2 u-1}+|\xi|^{-2 v-1} \sin ^{2}\left(|\xi|^{-w}\right),
$$

where the three parameters $u, v$ and $w$ are arbitrary real numbers such that $0<$ $u \leq v<1$ and $w>0$. Observe that the larger is $w$ the more oscillating is this function $f_{\mu}$ in the neighborhood of 0 . Also observe that this function fails to satisfy $\left(\mathcal{D}_{1, \mu}\right)$; yet, for any integer $M \geq 1+w$, it satisfies $\left(\mathcal{D}_{M}\right)$, with $\beta_{0}=u$ and $\beta_{0}^{\prime}=v$.

In the sequel, one only gives the proofs of Propositions 4.5 and 4.6. The other two Propositions can be derived rather similarly to Proposition 4.6.

Proof of Proposition 4.5: It easily follows from (2.14) and the equality $\psi:=\mathbf{1}_{[0,1 / 2)}-\mathbf{1}_{[1 / 2,1)}$ that, for all $j \in \mathbb{N}$ and $k \in\left\{-N_{1}, \ldots, 2^{j} v-N_{2}\right\}$, one has

$$
2^{-j / 2} \lambda_{j, k}=2 X\left(\frac{2 k+1}{2^{j+1}}\right)-X\left(\frac{k}{2^{j}}\right)-X\left(\frac{k+1}{2^{j}}\right) .
$$

Therefore, using (4.6) and standard computations, one gets, for any $k_{1}, k_{2} \in\left\{-N_{1}, \ldots, 2^{j} v-N_{2}\right\}$, that

$$
\mathbb{E}\left[\lambda_{j, k_{1}} \lambda_{j, k_{2}}\right]=2^{2(j+2)} \int_{\mathbb{R}} e^{i\left(k_{1}-k_{2}\right) \eta} \sin ^{4}\left(\frac{\eta}{4}\right) f_{X}\left(2^{j} \eta\right) \mathrm{d} \eta .
$$

There is no restriction to assume that $k_{1} \geq k_{2}$. Then, setting

$$
G(\eta):=e^{-i \eta} \sin ^{4}\left(\frac{\eta}{4}\right)
$$

one can write

$$
\mathbb{E}\left[\lambda_{j, k_{1}} \lambda_{j, k_{2}}\right]=2^{2(j+2)} \int_{\mathbb{R}} e^{i\left(1+\left|k_{1}-k_{2}\right|\right) \eta} F_{j}(\eta) \mathrm{d} \eta
$$

where

$$
F_{j}(\eta):=G(\eta) f_{X}\left(2^{j} \eta\right) .
$$

Next, observe that it easily results from (4.17), the general Leibniz rule and standard computations, that $G$ is infinitely differentiable on $\mathbb{R}$. Moreover, there is a finite deterministic constant $c_{1}>0$, such that, for all $q \in\left\{0, \ldots, V_{0}\right\}$ and $\eta \in \mathbb{R}$, one has

$$
\left|G^{(q)}(\eta)\right| \leq c_{1} \min \left(1,|\eta|^{4-q}\right),
$$

where $G^{(q)}$ denotes the derivative of $G$ of order $q$, with the convention that $G^{(0)}:=$ $G$.

Let us now derive useful properties of the function $F_{j}$ defined in (4.19). Notice that $F_{j}$ is $V_{0}$ times continuously differentiable on $\mathbb{R} \backslash\{0\}$ since $f_{X}$ is $V_{0}$ times continuously differentiable on $\mathbb{R} \backslash\{0\}$ and $G$ is infinitely differentiable on $\mathbb{R}$. Also notice that using (4.19), the general Leibniz rule, the condition $\left(\mathcal{D}_{1, X}\right)$ and (4.20), one can show that, for some finite deterministic constant $c_{2}>0$ (not depending on $j$ ) and for any arbitrary $\eta \in \mathbb{R} \backslash\{0\}$, one has, for all $p \in\left\{0, \ldots, V_{0}\right\}$,

$$
\left|F_{j}^{(p)}(\eta)\right| \leq c_{2} 2^{-j\left(2 \beta_{0}^{\prime}+1\right)}|\eta|^{3-p-2 \beta_{0}^{\prime}}, \quad \text { if } 0<|\eta|<2^{-j},
$$

and

$$
\left|F_{j}^{(p)}(\eta)\right| \leq c_{2} 2^{-j\left(2 \beta_{0}+1\right)} \sum_{q=0}^{p} \min \left(1,|\eta|^{4-q}\right)|\eta|^{q-2 \beta_{0}-p-1}, \quad \text { if }|\eta| \geq 2^{-j} .
$$


Next, observe that it follows from (4.21) and (4.22) that, for each $p \in\left\{0, \ldots, V_{0}\right\}$, the function $F_{j}^{(p)}$ belongs to the Lebesgue space $L^{1}(\mathbb{R})$ and has vanishing limits at $\pm \infty$. Moreover, it has a vanishing limit at 0 when $p \neq 2$.

In view of these properties of $F_{j}, V_{0}$ integrations by parts allow to obtain that

$$
\int_{0}^{+\infty} e^{i\left(1+\left|k_{1}-k_{2}\right|\right) \eta} F_{j}(\eta) \mathrm{d} \eta=i^{-V_{0}}\left(1+\left|k_{1}-k_{2}\right|\right)^{-V_{0}} \int_{0}^{+\infty} e^{i\left(1+\left|k_{1}-k_{2}\right|\right) \eta} F_{j}^{\left(V_{0}\right)}(\eta) \mathrm{d} \eta .
$$

Similarly one has

$$
\int_{-\infty}^{0} e^{i\left(1+\left|k_{1}-k_{2}\right|\right) \eta} F_{j}(\eta) \mathrm{d} \eta=i^{-V_{0}}\left(1+\left|k_{1}-k_{2}\right|\right)^{-V_{0}} \int_{-\infty}^{0} e^{i\left(1+\left|k_{1}-k_{2}\right|\right) \eta} F_{j}^{\left(V_{0}\right)}(\eta) \mathrm{d} \eta .
$$

Finally, putting together (4.18), (4.23), (4.24), (4.21) with $p=V_{0},(4.22)$ with $p=V_{0}$, and the inequality $\beta_{0}^{\prime} \geq \beta_{0}$, it can be shown that there exists a finite deterministic constant $c>0$, not depending on $j, k_{1}, k_{2}$, such that (4.10) holds.

In order to show that Proposition 4.6 holds one needs the following lemma, which provides an explicit convenient expression of the expectation $\mathbb{E}\left[\lambda_{j, k_{1}} \lambda_{j, k_{2}}\right]$ in terms of $f_{X}$ and of the Fourier transform $\widehat{\psi}$ of the wavelet $\psi$.

Lemma 4.10. Assume that the wavelet $\psi$ in (2.14) is continuously differentiable on the real line. Then, for all $j \in \mathbb{N}$ and for every $k_{1}, k_{2} \in\left\{-N_{1}, \ldots, 2^{j} v-N_{2}\right\}$, one has

$$
\mathbb{E}\left[\lambda_{j, k_{1}} \lambda_{j, k_{2}}\right]=2^{2 j} \int_{\mathbb{R}} e^{i\left(k_{1}-k_{2}\right) \eta} \eta^{2}|\widehat{\psi}(\eta)|^{2} f_{X}\left(2^{j} \eta\right) \mathrm{d} \eta .
$$

Proof of Lemma 4.10: First notice that the integration by parts formula can be applied to the Young integral in (2.14), since $\psi$ is continuously differentiable. Thus, in view of (2.8), one can express $\lambda_{j, k}$, for all $j \in \mathbb{N}$ and $k \in\left\{-N_{1}, \ldots, 2^{j} v-N_{2}\right\}$, as the Lebesgue integral

$$
\lambda_{j, k}=-2^{3 j / 2} \int_{\mathbb{R}} \psi^{\prime}\left(2^{j} s-k\right) X(s) \mathrm{d} s,
$$

where $\psi^{\prime}$ denotes the first order derivative of $\psi$. Then, using Fubini theorem, (4.6), elementary properties of the Fourier transform, and the change of variable $\eta=2^{-j} \xi$, one obtains

$$
\begin{aligned}
& \mathbb{E}\left[\lambda_{j, k_{1}} \lambda_{j, k_{2}}\right]=2^{3 j} \int_{\mathbb{R}} \int_{\mathbb{R}} \psi^{\prime}\left(2^{j} s_{1}-k_{1}\right) \psi^{\prime}\left(2^{j} s_{2}-k_{2}\right) \\
& \times \mathbb{E}\left[X\left(s_{1}\right) X\left(s_{2}\right)\right] \mathrm{d} s_{1} \mathrm{~d} s_{2} \\
& =2^{3 j} \int_{\mathbb{R}} \int_{\mathbb{R}} \int_{\mathbb{R}} \psi^{\prime}\left(2^{j} s_{1}-k_{1}\right) \psi^{\prime}\left(2^{j} s_{2}-k_{2}\right) \\
& \quad \times\left(e^{i s_{1} \xi}-1\right)\left(e^{-i s_{2} \xi}-1\right) f_{X}(\xi) \mathrm{d} \xi \mathrm{d} s_{1} \mathrm{~d} s_{2} \\
& =2^{-j} \int_{\mathbb{R}} e^{i\left(k_{1}-k_{2}\right) 2^{-j} \xi} \xi^{2}\left|\widehat{\psi}\left(2^{-j} \xi\right)\right|^{2} f_{X}(\xi) \mathrm{d} \xi \\
& =2^{2 j} \int_{\mathbb{R}} e^{i\left(k_{1}-k_{2}\right) \eta} \eta^{2}|\widehat{\psi}(\eta)|^{2} f_{X}\left(2^{j} \eta\right) \mathrm{d} \eta .
\end{aligned}
$$


Proof of Proposition 4.6: The proof is rather similar to that of Proposition 4.5. Let $j \in \mathbb{N}$ and $k_{1}, k_{2} \in\left\{-N_{1}, \ldots, 2^{j} v-N_{2}\right\}$ be arbitrary and fixed with $k_{1} \geq k_{2}$ (there is no restriction to assume this). In view of (4.25), setting

$$
\widetilde{G}(\eta):=e^{-i \eta} \eta^{2}|\widehat{\psi}(\eta)|^{2}
$$

$\mathbb{E}\left[\lambda_{j, k_{1}} \lambda_{j, k_{2}}\right]$ can then be expressed as

$$
\mathbb{E}\left[\lambda_{j, k_{1}} \lambda_{j, k_{2}}\right]=2^{2 j} \int_{\mathbb{R}} e^{i\left(1+\left|k_{1}-k_{2}\right|\right) \eta} \widetilde{F}_{j}(\eta) \mathrm{d} \eta,
$$

where

$$
\widetilde{F}_{j}(\eta):=\widetilde{G}(\eta) f_{X}\left(2^{j} \eta\right) .
$$

Now, let us derive the properties of the function $\widetilde{G}$ which one needs in the proof. First, observe that the fact that $\psi$ is compactly supported implies that $\widehat{\psi}$ is infinitely differentiable on $\mathbb{R}$. Thus, in view of (4.26), the function $\widetilde{G}$ shares the same regularity property. Next, we show that there is a finite deterministic constant $c_{1}>0$ such that the inequality

$$
\left|\widetilde{G}^{(q)}(\eta)\right| \leq c_{1} \min \left(1,|\eta|^{2(M+1)-q}\right)
$$

holds, for all $q \in\{0,1,2\}$ and $\eta \in \mathbb{R}$. In view of (4.26), the inequality (4.29) can be obtained by using the general Leibniz rule, if one shows that there exists a finite deterministic constant $c_{2}>0$ such that the inequality

$$
\left|\widehat{\psi}^{(n)}(\eta)\right| \leq c_{2} \min \left((1+|\eta|)^{-1},|\eta|^{M-n}\right)
$$

holds, for every $n \in\left\{0, \ldots, V_{0}\right\}$ and $\eta \in \mathbb{R}$. One can derive from the definition of the Fourier transform $\widehat{\psi}$ that, for any $l \in \mathbb{Z}_{+}$and $\eta \in \mathbb{R}$,

$$
\widehat{\psi}^{(l)}(\eta)=(-i)^{l} \int_{\mathbb{R}} e^{-i \eta n}\left(s^{l} \psi(s)\right) \mathrm{d} s .
$$

Notice that $s \mapsto s^{l} \psi(s)$ is a compactly supported continuously differentiable function. Therefore, an integration by parts in (4.31) yields, for each $\eta \in \mathbb{R}$, that

$$
\left|\widehat{\psi}^{(l)}(\eta)\right| \leq c_{3}(1+|\eta|)^{-1}
$$

where $c_{3}>0$ is a finite deterministic constant not depending on $\eta$. Thus, when $-1 \leq M-n \leq 0$, the inequality (4.30) results from the inequality (4.32). In the opposite case $M-n>0$, one knows from (4.13) and (4.31) that $\widehat{\psi}^{(n+r)}(0)=0$, for any nonnegative integer $r$ satisfying $r<M-n$. Thus, Taylor formula applied to $\widehat{\psi}^{(n)}$ allows to obtain, for all $\eta \in[-1,1]$, that

$$
\left|\widehat{\psi}^{(n)}(\eta)\right| \leq c_{4}|\eta|^{M-n}
$$

where $c_{4}>0$ is a finite deterministic constant not depending on $\eta$. Then combining (4.32) and (4.33) one gets (4.30).

Now, one derives some useful properties of the function $\widetilde{F}_{j}$ defined in (4.28). Notice that $\widetilde{F}_{j}$ is $V_{0}$ times continuously differentiable on $\mathbb{R} \backslash\{0\}$ since $f_{X}$ is $V_{0}$ times continuously differentiable on $\mathbb{R} \backslash\{0\}$ and $\widetilde{G}$ is infinitely differentiable on $\mathbb{R}$. Also notice that using (4.28), the general Leibniz rule, the condition $\left(\mathcal{D}_{M, X}\right)$ and (4.29), one can show that, for some finite deterministic constant $c_{5}>0$ (not depending on $j$ ) and for any arbitrary $\eta \in \mathbb{R} \backslash\{0\}$, one has, for all $p \in\left\{0, \ldots, V_{0}\right\}$,

$$
\left|\widetilde{F}_{j}^{(p)}(\eta)\right| \leq c_{5} 2^{-j\left(2 \beta_{0}^{\prime}+1\right)}|\eta|^{2 M+1-p M-2 \beta_{0}^{\prime}}, \text { if } 0<|\eta|<2^{-j},
$$


and

$$
\left|\widetilde{F}_{j}^{(p)}(\eta)\right| \leq c_{5} 2^{-j\left(2 \beta_{0}+1\right)} \sum_{q=0}^{p} \min \left(1,|\eta|^{2(M+1)-q}\right)|\eta|^{q-2 \beta_{0}-p-1}, \text { if }|\eta| \geq 2^{-j} .
$$

Next, observe that it follows from (4.34) and (4.35) that, for each $p \in\left\{0, \ldots, V_{0}\right\}$, the function $\widetilde{F}_{j}^{(p)}$ belongs to the Lebesgue space $L^{1}(\mathbb{R})$ and has vanishing limits at $\pm \infty$. Moreover, it has a vanishing limit at 0 when $p \neq 2$.

In view of these properties of $\widetilde{F}_{j}, V_{0}$ integrations by parts allow to obtain that

$$
\int_{0}^{+\infty} e^{i\left(1+\left|k_{1}-k_{2}\right|\right) \eta} \widetilde{F}_{j}(\eta) \mathrm{d} \eta=i^{-V_{0}}\left(1+\left|k_{1}-k_{2}\right|\right)^{-V_{0}} \int_{0}^{+\infty} e^{i\left(1+\left|k_{1}-k_{2}\right|\right) \eta} \widetilde{F}_{j}^{\left(V_{0}\right)}(\eta) \mathrm{d} \eta .
$$

Similarly, one has that

$$
\int_{-\infty}^{0} e^{i\left(1+\left|k_{1}-k_{2}\right|\right) \eta} \widetilde{F}_{j}(\eta) \mathrm{d} \eta=i^{-V_{0}}\left(1+\left|k_{1}-k_{2}\right|\right)^{-V_{0}} \int_{-\infty}^{0} e^{i\left(1+\left|k_{1}-k_{2}\right|\right) \eta} \widetilde{F}_{j}^{\left(V_{0}\right)}(\eta) \mathrm{d} \eta .
$$

Finally, putting together (4.27), (4.36), (4.37), (4.34) with $p=V_{0}$, (4.35) with $p=V_{0}$, and the inequality $\beta_{0}^{\prime} \geq \beta_{0}$, it can be shown that there exists a finite deterministic constant $c>0$, not depending on $j, k_{1}, k_{2}$, such that (4.10) holds.

\section{Discussion on the optimality of the improved rate of convergence}

What is for the approximation error $\left\|Y-Y_{J}^{W}\right\|_{C^{\gamma}(I)}$ the fastest possible rate of convergence to zero? In order to provide a precise answer to this question, let us first briefly summarize what is already known on it from our previous two main results, namely Theorems 2.3 and 3.5. To this end, one denotes by $\bar{\alpha}$ and $\bar{\beta}$ the two critical exponents defined as:

$$
\bar{\alpha}:=\sup \left\{\alpha \in[0,1): \sigma \in C^{\alpha}(I)\right\} \text { and } \bar{\beta}:=\sup \left\{\beta \in[0,1): X \in C^{\beta}(I)\right\} \text {. }
$$

They somehow respectively provide a measure of the critical Hölder regularity on $I$ of a path of the integrand $\sigma$ and a measure of that of a path of the integrator $X$. Notice that the assumption on the Hölder regularity of these two paths, made at the very beginning of the article, implies that:

$$
\bar{\alpha} \in(0,1], \quad \bar{\beta} \in(0,1] \text { and } \bar{\alpha}+\bar{\beta}>1 .
$$

In the sequel, one imposes to $\bar{\alpha}$ and $\bar{\beta}$ to satisfy the following invariance condition $(\mathcal{U C})$.

Definition 5.1 (the invariance condition $(\mathcal{U C})$ ). The values of $\bar{\alpha}$ and $\bar{\beta}$ remain almost surely unchanged when the compact interval $I:=[0, v]$ in $(5.1)$ is replaced by any other compact interval $\mathcal{K} \subset \mathbb{R}$.

It is worth mentioning that the condition $(\mathcal{U C})$ holds when $\sigma$ and $X$ are Gaussian fractional Brownian motions; in this case $\bar{\alpha}$ and $\bar{\beta}$ are equal to their corresponding Hurst parameters.

Remark 5.2. Under the condition $(\mathcal{U C})$, Theorem 2.3 can be rewritten in terms of $\bar{\alpha}$ and $\bar{\beta}$ in the following way: for each fixed $\gamma \in[0, \bar{\beta})$ and arbitrarily small $\epsilon>0$, 
one has

$$
\sup _{J \in \mathbb{N}}\left\{2^{J(\min (\bar{\beta}-\gamma, \bar{\alpha}+\bar{\beta}-1)-\epsilon)}\left\|Y-Y_{J}^{W}\right\|_{C^{\gamma}(I)}\right\}<+\infty .
$$

Remark 5.3. Assume that the condition $(\mathcal{U C})$ holds. Also, assume that the condition $(\mathcal{W} C)$, described in Definition 3.3, is satisfied for all $\alpha_{0} \in(0, \bar{\alpha})$ and $\beta_{0} \in(0, \bar{\beta})$ such that $\alpha_{0}+\beta_{0}>1$. Then, Theorem 3.5 can be rewritten in terms of $\bar{\alpha}$ and $\bar{\beta}$ in the following way: for each fixed $\gamma \in[0, \min (\bar{\beta}, 1 / 2))$ and arbitrarily small $\epsilon>0$, one has, almost surely,

$$
\sup _{J \in \mathbb{N}}\left\{2^{J(\min (\bar{\beta}-\gamma, \bar{\alpha}+\bar{\beta}-1 / 2-\gamma)-\epsilon)}\left\|Y-Y_{J}^{W}\right\|_{C^{\gamma}(I)}\right\}<+\infty .
$$

Let us now turn to the statement of the main result of the present section.

Theorem 5.4. Suppose that the invariance condition $(\mathcal{U C})$ holds. Also, suppose that $\bar{\beta}<1$ and that, for each $s \in I$, the probability $\mathbb{P}(\sigma(s)=0$ ) vanishes (notice that this is the case as soon as the random variable $\sigma(s)$ has a probability density function). Then for all fixed $\gamma \in[0, \bar{\beta})$ and arbitrarily small $\epsilon>0$, one has, almost surely,

$$
\sup _{J \in \mathbb{N}}\left\{2^{J(\bar{\beta}-\gamma+\epsilon)}\left\|Y-Y_{J}^{W}\right\|_{C^{\gamma}(I)}\right\}=+\infty .
$$

We point out that Theorem 5.4 provides an universal lower bound for the rate of convergence to zero of the approximation error $\left\|Y-Y_{J}^{W}\right\|_{C^{\gamma}(I)}$. In view of Remarks 5.2 and 5.3, this theorem can be seen as a counterpart to Theorems 2.3 and 3.5.

Before proving Theorem 5.4, we note that the following result, which is a straightforward consequence of Theorems 5.4 and 3.5 (see also Remark 5.3), gives a partial answer to the question raised at the very beginning of the present section.

Corollary 5.5. Assume that the invariance condition $(\mathcal{U C})$ holds and that $\bar{\alpha} \geq 1 / 2$ and $\bar{\beta}<1$. Also assume that the condition $(\mathcal{W} C)$, given by Definition 3.3, is satisfied for all $\alpha_{0} \in(0, \bar{\alpha})$ and $\beta_{0} \in(0, \bar{\beta})$ such that $\alpha_{0}+\beta_{0}>1$. Moreover, suppose that, for any $s \in I$, the probability $\mathbb{P}(\sigma(s)=0)$ vanishes. Then, for each fixed $\gamma \in[0, \min (\bar{\beta}, 1 / 2))$ and arbitrarily small $\epsilon>0$, one has, almost surely,

$$
\sup _{J \in \mathbb{N}}\left\{2^{J(\bar{\beta}-\gamma-\epsilon)}\left\|Y-Y_{J}^{W}\right\|_{C^{\gamma}(I)}\right\}<+\infty
$$

and

$$
\sup _{J \in \mathbb{N}}\left\{2^{J(\bar{\beta}-\gamma+\epsilon)}\left\|Y-Y_{J}^{W}\right\|_{C^{\gamma}(I)}\right\}=+\infty,
$$

which provide a sharp estimate of the fastest possible rate of convergence to zero of the approximation error $\left\|Y-Y_{J}^{W}\right\|_{C^{\gamma}(I)}$. In the sequel, one uses the concise notation

$$
\left\|Y-Y_{J}^{W}\right\|_{C^{\gamma}(I)} \asymp 2^{-J(\bar{\beta}-\gamma)}
$$

to mean that both (5.5) and (5.6) are satisfied, almost surely, for all fixed arbitrarily small $\epsilon>0$.

Remark 5.6. Assume that the integrand $\sigma$ and the integrator $X$ are two independent Gaussian fractional Brownian motions whose Hurst parameters are respectively denoted by $H_{1}$ and $H_{2}$ and satisfy $H_{1} \geq 1 / 2, H_{1}+H_{2}>1$.

Then, it results from Corollary 5.5 that, for all fixed $\gamma \in\left[0, \min \left(H_{2}, 1 / 2\right)\right)$, one has $\left\|Y-Y_{J}^{W}\right\|_{C^{\gamma}(I)} \asymp 2^{-J\left(H_{2}-\gamma\right)}$. 
Proof of Theorem 5.4: First observe that using the second equality in (5.1), Proposition 1.1, the condition $(\mathcal{U C})$ and the assumption that, for all $s \in I$, the probability $\mathbb{P}(\sigma(s)=0)$ vanishes, one has

$$
\bar{\beta}=\sup \left\{\theta \in[0,1): Y \in C^{\theta}(I)\right\} .
$$

In order to show that (5.4) holds one will argue by contradiction. So, let us assume that there exist $\epsilon_{0} \in(0,1-\bar{\beta})$ and $\gamma_{0} \in[0, \bar{\beta})$ such that

$$
\sup _{J \in \mathbb{N}}\left\{2^{J\left(\bar{\beta}-\gamma_{0}+\epsilon_{0}\right)}\left\|Y-Y_{J}^{W}\right\|_{C^{\gamma_{0}(I)}}\right\}<+\infty .
$$

Under this assumption one will show that

$$
\bar{\beta}<\sup \left\{\theta \in[0,1): Y \in C^{\theta}(I)\right\},
$$

which will contradict (5.7).

Let $\nu_{0}$ be an arbitrary and fixed real number satisfying

$$
0<\nu_{0}<\frac{\epsilon_{0}}{\bar{\beta}-\gamma_{0}} .
$$

Observe that (5.10) implies that

$$
\left(\bar{\beta}+\gamma_{0} \nu_{0}+\epsilon_{0}\right)\left(1+\nu_{0}\right)^{-1}>\bar{\beta} .
$$

Denote by $t_{1}$ and $t_{2}$ two arbitrary real numbers belonging to $I$ with $t_{1}<t_{2}$. Then there is a unique positive integer $\breve{J}$ such that

$$
v 2^{-\breve{J}\left(1+\nu_{0}\right)}<t_{2}-t_{1} \leq v 2^{-(\breve{J}-1)\left(1+\nu_{0}\right)} .
$$

It follows from (1.2), (5.8) and (5.12) that

$$
\begin{aligned}
& \left|\left(Y-Y_{J}^{W}\right)\left(t_{2}\right)-\left(Y-Y_{J}^{W}\right)\left(t_{1}\right)\right| \leq\left\|Y-Y_{J}^{W}\right\|_{C^{\gamma_{0}(I)}}\left(t_{2}-t_{1}\right)^{\gamma_{0}} \\
& \leq c_{1} 2^{-\breve{J}\left(\bar{\beta}-\gamma_{0}+\epsilon_{0}\right)}\left(t_{2}-t_{1}\right)^{\gamma_{0}} \leq c_{2}\left(t_{2}-t_{1}\right)^{\left(\bar{\beta}+\gamma_{0} \nu_{0}+\epsilon_{0}\right)\left(1+\nu_{0}\right)^{-1}},
\end{aligned}
$$

where $c_{1}>0$ and $c_{2}>0$ are two finite random constants not depending on $t_{1}, t_{2}$ and $\check{J}$.

Next, one assumes that $\epsilon_{1}$ is an arbitrary fixed real number satisfying

$$
0<\epsilon_{1}<\min \left(\bar{\beta},(1-\bar{\beta}) \nu_{0}\right) .
$$

Observe that (5.14) implies that

$$
\left(\bar{\beta}+\nu_{0}-\epsilon_{1}\right)\left(1+\nu_{0}\right)^{-1}>\bar{\beta} .
$$

Let us show that there exists a finite random constant $c_{3}>0$, not depending on $t_{1}, t_{2}$ and $\breve{J}$, such that

$$
\left|Y_{\bar{J}}^{W}\left(t_{2}\right)-Y_{\bar{J}}^{W}\left(t_{1}\right)\right| \leq c_{3}\left(t_{2}-t_{1}\right)^{\left(\bar{\beta}+\nu_{0}-\epsilon_{1}\right)\left(1+\nu_{0}\right)^{-1}} .
$$

It follows from (2.11), (2.13), (2.15), the triangle inequality, and property $\left(\mathcal{P}_{1}\right)$ in Lemma 2.6 that

$$
\begin{aligned}
& \left|Y_{\breve{J}}^{W}\left(t_{2}\right)-Y_{\breve{J}}^{W}\left(t_{1}\right)\right| \leq \sum_{l=1-N_{2}}^{2^{\breve{J}} v-N_{1}}\left|b_{\breve{J}, l}\left(t_{2}\right)-b_{\breve{J}, l}\left(t_{1}\right)\right|\left|\eta_{\breve{J}, l}\right| \\
& \leq c_{4} 2^{\breve{J}\left(1+\epsilon_{1}-\bar{\beta}\right)} \sum_{l=1-N_{2}}^{2^{\breve{J}} v-N_{1}}\left|\int_{t_{1}}^{t_{2}} \sigma(s) \varphi\left(2^{\breve{J}} s-l\right) \mathrm{d} s\right|,
\end{aligned}
$$


where $c_{4}>0$ is a finite random constant depending on $\epsilon_{1}$ but not depending on $t_{1}$, $t_{2}$ and $\breve{J}$ (in fact $c_{4}$ is nothing else than the constant $c_{1}$ in (2.19) in the case where $\beta=\bar{\beta}-\epsilon_{1}$ ). Next, observe that, using (5.12), it can be shown that the cardinality of the finite set

$$
\left\{l \in\left\{1-N_{2}, \ldots, 2^{\breve{J}} v-N_{1}\right\}:\left[\frac{l+N_{1}}{2^{\breve{J}}}, \frac{l+N_{2}}{2^{\breve{J}}}\right] \cap\left[t_{1}, t_{2}\right] \neq \emptyset\right\}
$$

can be bounded by some finite deterministic constant $c_{5}>0$ not depending on $t_{1}$, $t_{2}$ and $\check{J}$. Thus setting $c_{6}:=c_{4} c_{5}\|\sigma\|_{\left[N_{1}, N_{2}\right], \infty}\|\varphi\|_{\left[N_{1}, N_{2}\right], \infty}$, where the integers $N_{1}$ and $N_{2}$ are as in (2.8), one can derive from (1.3) and (5.17) that

$$
\left|Y_{J}^{W}\left(t_{2}\right)-Y_{J}^{W}\left(t_{1}\right)\right| \leq c_{6} 2^{\check{J}\left(1+\epsilon_{1}-\bar{\beta}\right)}\left(t_{2}-t_{1}\right) .
$$

Then combining (5.12) and (5.18) one gets (5.16).

Finally, putting together (5.11), (5.13), (5.15) and (5.16), one shows that (5.9) holds, which contradicts (5.7).

\section{Appendix}

Proof of Proposition 1.4: First notice that using (1.5) as well as the fact that the paths of $X$ satisfy a Hölder condition of order $\beta$ on $I:=[0, v]$, and setting $c_{1}:=$ $\|X\|_{C^{\beta}(I)}$, one has that

$$
\left|\Delta_{J, k}(X)\right| \leq c_{1} 2^{-\beta J}, \quad \text { for every } J \in \mathbb{N} \text { and } k \in\left\{0, \ldots, 2^{J} v-1\right\} .
$$

From now on, one denotes by $t_{1}$ and $t_{2}$ two arbitrary fixed real numbers belonging to $I$ and satisfying $t_{1}<t_{2}$. In view of Remark 1.2, one has that

$$
\left|Y\left(t_{2}\right)-Y\left(t_{1}\right)\right| \leq c_{2}\left(t_{2}-t_{1}\right)^{\beta},
$$

where $c_{2}>0$ is a finite random constant not depending on $t_{1}$ and $t_{2}$.

Next, for each $\gamma \in[0, \beta)$ and $J \in \mathbb{N}$, one sets

$$
\mathcal{E}_{J}^{(\gamma)}\left(t_{1}, t_{2}\right):=\frac{\left|Y\left(t_{2}\right)-Y_{J}^{R S}\left(t_{2}\right)-Y\left(t_{1}\right)+Y_{J}^{R S}\left(t_{1}\right)\right|}{\left(t_{2}-t_{1}\right)^{\gamma}} .
$$

In view of (1.2), in order to show that (1.10) holds, one has to prove that there exists a finite random constant $c_{3}>0$, not depending on $\gamma, t_{1}, t_{2}$ and $J$, such that

$$
\mathcal{E}_{J}^{(\gamma)}\left(t_{1}, t_{2}\right) \leq c_{3} 2^{-J \min (\beta-\gamma, \alpha+\beta-1)} .
$$

To this end, one denotes by $k_{1}$ and $k_{2}$ the two integers, belonging to $\left\{0, \ldots, 2^{J} v\right\}$, defined as $k_{1}:=\left[2^{J} t_{1}\right]$ and $k_{2}:=\left[2^{J} t_{2}\right]$, and studies the three different cases: $k_{1}=k_{2}, k_{1}+1=k_{2}$ and $k_{1}+2 \leq k_{2}$.

First case: $k_{1}=k_{2}$. It follows from (1.6), (1.3) and (5.19) that

$$
\begin{aligned}
\left|Y_{J}^{R S}\left(t_{2}\right)-Y_{J}^{R S}\left(t_{1}\right)\right| & =\left|2^{J}\left(t_{2}-t_{1}\right) \sigma\left(\tilde{s}_{J, k_{1}}\right) \Delta_{J, k_{1}}(X)\right| \\
& \leq c_{1}\left(t_{2}-t_{1}\right)\|\sigma\|_{I, \infty} 2^{(1-\beta) J}
\end{aligned}
$$

On the other hand, one necessarily has that $t_{2}-t_{1}<2^{-J}$ since $k_{1}=k_{2}$. Combining this inequality with (5.23), (5.21), the triangle inequality and (5.20), one obtains

$$
\begin{aligned}
\mathcal{E}_{J}^{(\gamma)}\left(t_{1}, t_{2}\right) & \leq c_{2}\left(t_{2}-t_{1}\right)^{\beta-\gamma}+c_{1}\left(t_{2}-t_{1}\right)^{1-\gamma}\|\sigma\|_{I, \infty} 2^{(1-\beta) J} \\
& \leq c_{4} 2^{-J(\beta-\gamma)}
\end{aligned}
$$

where $c_{4}>0$ is a finite random constant not depending on $\gamma, J, t_{1}$ and $t_{2}$. 
Second case: $k_{1}+1=k_{2}$. First one notes that this case is rather similar to the previous one. It follows from (1.6) and (1.4) that

$$
\begin{aligned}
& Y_{J}^{R S}\left(t_{2}\right)-Y_{J}^{R S}\left(t_{1}\right) \\
& =\left(2^{J} t_{2}-k_{1}-1\right) \sigma\left(\tilde{s}_{J, k_{1}+1}\right) \Delta_{J, k_{1}+1}(X)+Y_{J}\left(\frac{k_{1}+1}{2^{J}}\right) \\
& \quad-\left(2^{J} t_{1}-k_{1}\right) \sigma\left(\tilde{s}_{J, k_{1}}\right) \Delta_{J, k_{1}}(X)-Y_{J}\left(\frac{k_{1}}{2^{J}}\right) \\
& =\left(2^{J} t_{2}-k_{1}-1\right) \sigma\left(\tilde{s}_{J, k_{1}+1}\right) \Delta_{J, k_{1}+1}(X) \\
& \quad+\left(k_{1}+1-2^{J} t_{1}\right) \sigma\left(\tilde{s}_{J, k_{1}}\right) \Delta_{J, k_{1}}(X) .
\end{aligned}
$$

Thus, using the triangle inequality, (5.19) and (1.3) we obtain that

$$
\begin{aligned}
& \left|Y_{J}^{R S}\left(t_{2}\right)-Y_{J}^{R S}\left(t_{1}\right)\right| \\
& \leq c_{1}\left(2^{J} t_{2}-k_{1}-1\right)\|\sigma\|_{I, \infty} 2^{-\beta J}+c_{1}\left(k_{1}+1-2^{J} t_{1}\right)\|\sigma\|_{I, \infty} 2^{-\beta J} \\
& =c_{1}\left(t_{2}-t_{1}\right)\|\sigma\|_{I, \infty} 2^{(1-\beta) J} .
\end{aligned}
$$

On the other hand, it is necessarily true that $t_{2}-t_{1}<2 \cdot 2^{-J}$, since $k_{1}+1=k_{2}$. Combining this inequality with (5.25), (5.21), the triangle inequality and (5.20), we get that

$$
\mathcal{E}_{J}^{(\gamma)}\left(t_{1}, t_{2}\right) \leq c_{5} 2^{-J(\beta-\gamma)},
$$

where $c_{5}>0$ is a finite random constant not depending on $\gamma, J, t_{1}$ and $t_{2}$.

Third case: $k_{1}+2 \leq k_{2}$. It follows from (1.1), the triangle inequality and (5.20) that

$$
\begin{aligned}
& \left|Y\left(t_{2}\right)-Y_{J}^{R S}\left(t_{2}\right)-Y\left(t_{1}\right)+Y_{J}^{R S}\left(t_{1}\right)\right| \\
& \leq\left|Y\left(t_{2}\right)-Y\left(\frac{k_{2}}{2^{J}}\right)\right|+\left|Y\left(t_{1}\right)-Y\left(\frac{k_{1}}{2^{J}}\right)\right| \\
& \quad+\left|\int_{\frac{k_{1}}{2^{J}}}^{\frac{k_{2}}{2^{J}}} \sigma(s) \mathrm{d} X(s)-Y_{J}^{R S}\left(t_{2}\right)+Y_{J}^{R S}\left(t_{1}\right)\right| \\
& \leq 2 c_{2} 2^{-\beta J}+\left|\int_{\frac{k_{1}}{2^{J}}}^{\frac{k_{2}}{2^{J}}} \sigma(s) \mathrm{d} X(s)-Y_{J}^{R S}\left(t_{2}\right)+Y_{J}^{R S}\left(t_{1}\right)\right| .
\end{aligned}
$$

Moreover, using (1.6), the triangle inequality, (1.3), (1.4), (5.19), (1.5) and (1.8), one has that

$$
\begin{aligned}
& \left|\int_{\frac{k_{1}}{2^{J}}}^{\frac{k_{2}}{2^{J}}} \sigma(s) \mathrm{d} X(s)-Y_{J}^{R S}\left(t_{2}\right)+Y_{J}^{R S}\left(t_{1}\right)\right| \\
& \leq\left|\int_{\frac{k_{1}}{2^{J}}}^{\frac{k_{2}}{2^{J}}} \sigma(s) \mathrm{d} X(s)-Y_{J}^{R S}\left(\frac{k_{2}}{2^{J}}\right)+Y_{J}^{R S}\left(\frac{k_{1}}{2^{J}}\right)\right| \\
& +\left|\left(2^{J} t_{2}-k_{2}\right) \sigma\left(\tilde{s}_{J, k_{2}}\right) \Delta_{J, k_{2}}(X)\right|+\left|\left(2^{J} t_{1}-k_{1}\right) \sigma\left(\tilde{s}_{J, k_{1}}\right) \Delta_{J, k_{1}}(X)\right| \\
& \leq \sum_{l=k_{1}}^{k_{2}-1}\left|\int_{\frac{l}{2^{J}}}^{\frac{l+1}{2^{J}}} \sigma(s) \mathrm{d} X(s)-\sigma\left(\tilde{s}_{J, l}\right) \Delta_{J, l}(X)\right|+2 c_{1}\|\sigma\|_{I, \infty} 2^{-\beta J} \\
& \leq c_{6}\left(k_{2}-k_{1}\right) 2^{-(\alpha+\beta) J}+2 c_{1}\|\sigma\|_{I, \infty} 2^{-\beta J} \\
& \leq c_{7}\left(t_{2}-t_{1}\right) 2^{-(\alpha+\beta-1) J}+2 c_{1}\|\sigma\|_{I, \infty} 2^{-\beta J},
\end{aligned}
$$


where $c_{6}>0$ and $c_{7}>0$ are two finite random constants not depending on $J$, $t_{1}$ and $t_{2}$. On the other hand, the fact that $k_{1}+2 \leq k_{2}$ yields $t_{2}-t_{1}>2^{-J}$. Combining this inequality with (5.27), (5.28) and (5.21), one gets

$$
\mathcal{E}_{J}^{(\gamma)}\left(t_{1}, t_{2}\right) \leq 2\left(c_{2}+c_{1}\|\sigma\|_{I, \infty}\right) 2^{-(\beta-\gamma) J}+c_{7}\left(t_{2}-t_{1}\right)^{1-\gamma} 2^{-(\alpha+\beta-1) J} .
$$

Thus, it results from the inequality $\left(t_{2}-t_{1}\right)^{1-\gamma} \leq v^{1-\gamma}$ that

$$
\mathcal{E}_{J}^{(\gamma)}\left(t_{1}, t_{2}\right) \leq c_{8} 2^{-J \min (\beta-\gamma, \alpha+\beta-1)},
$$

where $c_{8}>0$ is a finite random constant not depending on $\gamma, J, t_{1}$ and $t_{2}$.

Finally (5.24), (5.26) and (5.29) imply that (5.22) holds.

Let us now turn to prove Lemma 2.6. In the proof one will separately study the case of the Haar basis (see $\left(\mathcal{H}_{1}\right)$ at the very beginning of Section 2 ) and the other case where the compactly supported scaling function $\varphi$ and mother wavelet $\psi$ are $\alpha$-Hölder continuous on $\mathbb{R}$ (see $\left(\mathcal{H}_{2}\right)$ at the very beginning of Section 2 ).

Proof of Lemma 2.6 under the hypothesis $\left(\mathcal{H}_{1}\right)$ : First notice that, under the hypothesis $\left(\mathcal{H}_{1}\right)$, the integers $N_{1}$ and $N_{2}$ in (2.8) can be chosen such that $N_{1}=0$ and $N_{2}=1$, since one has

$$
\varphi:=\mathbf{1}_{[0,1)} \quad \text { and } \quad \psi:=\mathbf{1}_{[0,1 / 2)}-\mathbf{1}_{[1 / 2,1)} .
$$

In all the sequel, one assumes that $J \in \mathbb{N}$ and $l \in\left\{0, \ldots, 2^{J} v-1\right\}$ are arbitrary and fixed, and denotes by $\mathcal{I}_{J, l}$ the dyadic interval $\left[2^{-J} l, 2^{-J}(l+1)\right]$. Recall that $I:=[0, v]$.

Let us show that $\left(\mathcal{P}_{1}\right)$ is satisfied. Notice that, in view of $(5.30)$, one has that

$$
\int_{2^{-J}\left(l+N_{1}\right)}^{2^{-J}\left(l+N_{2}\right)} \varphi\left(2^{J} s-l\right) \mathrm{d} X(s)=\int_{2^{-J} l}^{2^{-J}(l+1)} \mathrm{d} X(s)=X\left(2^{-J}(l+1)\right)-X\left(2^{-J} l\right) .
$$

Thus, the fact that the paths of $X$ are Hölder continuous of order $\beta$ on the interval $I$ implies that (2.19) holds. Using rather similar arguments, it can be shown that $\left(\mathcal{P}_{1}^{\prime}\right)$ is satisfied as well.

Let us now turn to $\left(\mathcal{P}_{2}\right)$. Notice that, in view of (5.30) and (2.22) one has that

$$
\begin{aligned}
& \int_{2^{-J}\left(l+N_{1}\right)}^{2^{-J}\left(l+N_{2}\right)}\left(\sigma(s)-\bar{\sigma}_{J, l}\right) \varphi\left(2^{J} s-l\right) \mathrm{d} X(s) \\
& =\int_{2^{-J} l}^{2^{-J}(l+1)} \sigma(s) \mathrm{d} X(s)-\bar{\sigma}_{J, l}\left(X\left(2^{-J}(l+1)\right)-X\left(2^{-J} l\right)\right),
\end{aligned}
$$

where

$$
\bar{\sigma}_{J, l}=2^{J} \int_{2^{-J} l}^{2^{-J}(l+1)} \sigma(s) \mathrm{d} s
$$

Also notice that, using the continuity of the paths of $\sigma$ and the mean value theorem, one has $\bar{\sigma}_{J, l}=\sigma\left(\tilde{s}_{J, l}\right)$, for some $\tilde{s}_{J, l} \in \mathcal{I}_{J, l}$. Thus, combining this equality with (5.31), Remark 1.3, the inequality $\|\sigma\|_{C^{\alpha}\left(\mathcal{I}_{J, l}\right)} \leq\|\sigma\|_{C^{\alpha}(I)}$ and the inequality $\|X\|_{C^{\beta}\left(\mathcal{I}_{J, l}\right)} \leq\|X\|_{C^{\beta}(I)}$, one gets (2.21).

Finally, let us turn to $\left(\mathcal{P}_{3}\right)$. Assume that $t_{1}, t_{2} \in I$ are arbitrary and such that $t_{1}<t_{2}$. Also assume that $l \in \partial \mathbb{L}_{J, t_{1}, t_{2}}$ (see (2.17)), and let $[a, b]$ be the nonempty interval defined as

$$
[a, b]:=\mathcal{I}_{J, l} \cap\left[t_{1}, t_{2}\right]=\left[2^{-J} l, 2^{-J}(l+1)\right] \cap\left[t_{1}, t_{2}\right] .
$$


Then, in view of (5.30), one has

$$
\int_{t_{1}}^{t_{2}} \sigma(s) \varphi\left(2^{J} s-l\right) \mathrm{d} X(s)=\int_{a}^{b} \sigma(s) \mathrm{d} X(s) .
$$

Moreover, using the triangle inequality, Proposition 1.1, the fact that the paths of $X$ are $\beta$-Hölder continuous functions on $I,(1.3)$ and (5.32), one gets that

$$
\begin{aligned}
& \left|\int_{a}^{b} \sigma(s) \mathrm{d} X(s)\right| \\
& \leq\left|\int_{a}^{b} \sigma(s) \mathrm{d} X(s)-\sigma(a)(X(b)-X(a))\right|+|\sigma(a)(X(b)-X(a))| \\
& \leq c_{4} \min \left(2^{-\beta J},\left(t_{2}-t_{1}\right)^{\beta}\right),
\end{aligned}
$$

where $c_{4}:=\mathcal{K}_{\alpha+\beta}\|\sigma\|_{C^{\alpha}(I)}\|X\|_{C^{\beta}(I)}+\|\sigma\|_{I, \infty}\|X\|_{C^{\beta}(I)}$. Thus (2.23) results from (5.33) and (5.34).

Proof of Lemma 2.6 under the hypothesis $\left(\mathcal{H}_{2}\right)$ : Throughout this proof, one assumes that $J \in \mathbb{N}$ and $l \in\left\{1-N_{2}, \ldots, 2^{J} v-N_{1}\right\}$ are arbitrary and fixed and denotes by $\mathcal{I}_{J, l}$ the dyadic interval $\left[2^{-J}\left(l+N_{1}\right), 2^{-J}\left(l+N_{2}\right)\right]$; notice that this is not exactly the same interval as in the previous proof.

Let us show that $\left(\mathcal{P}_{1}\right)$ is satisfied. Using Proposition 1.1 and the equality $\varphi\left(N_{1}\right)=0($ see $(2.8))$, one gets that

$$
\begin{aligned}
& \left|\int_{2^{-J}\left(l+N_{1}\right)}^{2^{-J}\left(l+N_{2}\right)} \varphi\left(2^{J} s-l\right) \mathrm{d} X(s)\right| \\
& \quad \leq \mathcal{K}_{\alpha+\beta}\left(N_{2}-N_{1}\right)^{\alpha+\beta}\left\|\varphi\left(2^{J} \bullet-l\right)\right\|_{C^{\alpha}\left(\mathcal{I}_{J, l}\right)}\|X\|_{C^{\beta}\left(\mathcal{I}_{J, l}\right)} 2^{-(\alpha+\beta) J} .
\end{aligned}
$$

Moreover, it can be derived from (1.2) that

$$
\left\|\varphi\left(2^{J} \bullet-l\right)\right\|_{C^{\alpha}\left(\mathcal{I}_{J, l}\right)} \leq 2^{J \alpha}\|\varphi\|_{C^{\alpha}\left(\left[N_{1}, N_{2}\right]\right)}
$$

Also, it can be derived from (1.2) and (2.12) that

$$
\|X\|_{C^{\beta}\left(\mathcal{I}_{J, l}\right)} \leq\|X\|_{C^{\beta}\left(\left[Q_{1}, Q_{2}\right]\right)}
$$

Thus, putting together (5.35), (5.36) and (5.37), it follows that (2.19) holds. Using rather similar arguments, it can be shown that $\left(\mathcal{P}_{1}^{\prime}\right)$ is satisfied as well.

Let us now turn to $\left(\mathcal{P}_{2}\right)$. One can derive from the triangle inequality, (1.2), (1.3), (5.36) and (5.37) that

$$
\begin{aligned}
& \left\|\left(\sigma(\bullet)-\bar{\sigma}_{J, l}\right) \varphi\left(2^{J} \bullet-l\right)\right\|_{C^{\alpha}\left(\mathcal{I}_{J, l}\right)} \\
& \begin{array}{l}
\leq\left\|\sigma-\bar{\sigma}_{J, l}\right\|_{\mathcal{I}_{J, l}, \infty}\|\varphi\|_{\left[N_{1}, N_{2}\right], \infty}+\left\|\sigma-\bar{\sigma}_{J, l}\right\|_{\mathcal{I}_{J, l}, \infty}\left\|\varphi\left(2^{J} \bullet-l\right)\right\|_{C^{\alpha}\left(\mathcal{I}_{J, l}\right)} \\
\quad+\|\sigma\|_{C^{\alpha}\left(\mathcal{I}_{J, l}\right)}\|\varphi\|_{\left[N_{1}, N_{2}\right], \infty}
\end{array} \\
& \leq c_{4}\left(\left(2^{J \alpha}+1\right)\left\|\sigma-\bar{\sigma}_{J, l}\right\|_{\mathcal{I}_{J, l}, \infty}+\|\sigma\|_{C^{\alpha}\left(\left[Q_{1}, Q_{2}\right]\right)}\right)
\end{aligned}
$$


where $c_{4}:=\|\varphi\|_{C^{\alpha}\left(\left[N_{1}, N_{2}\right]\right)}$. Next, using (2.22), in which one sets $u=2^{J} s-l$, and using (2.2), one gets, for all fixed $x \in \mathcal{I}_{J, l}$, that

$$
\begin{aligned}
\left|\sigma(x)-\bar{\sigma}_{J, l}\right| & =\left|\int_{-\infty}^{+\infty}\left(\sigma(x)-\sigma\left(2^{-J} l+2^{-J} u\right)\right) \varphi(u) \mathrm{d} u\right| \\
& \leq \int_{N_{1}}^{N_{2}}\left|\sigma(x)-\sigma\left(2^{-J} l+2^{-J} u\right)\right||\varphi(u)| \mathrm{d} u \\
& \leq c_{5} 2^{-J \alpha}
\end{aligned}
$$

where $c_{5}:=\left(N_{2}-N_{1}\right)^{\alpha}\|\sigma\|_{C^{\alpha}\left(\left[Q_{1}, Q_{2}\right]\right)} \int_{-N}^{N}|\varphi(u)| \mathrm{d} u$. Therefore, in view of (1.3), one has

$$
\left\|\sigma-\bar{\sigma}_{J, l}\right\|_{\mathcal{I}_{J, l}, \infty} \leq c_{5} 2^{-J \alpha} .
$$

Next putting together (5.37), (5.38) and (5.39), it follows that there is a finite random constant $c_{6}>0$, not depending on $J, l$, such that

$$
\left\|\left(\sigma(\bullet)-\bar{\sigma}_{J, l}\right) \varphi\left(2^{J} \bullet-l\right)\right\|_{C^{\alpha}\left(\mathcal{I}_{J, l}\right)}\|X\|_{C^{\beta}\left(\mathcal{I}_{J, l}\right)} \leq c_{6} .
$$

Thus, using Proposition 1.1 and the equality $\varphi\left(N_{1}\right)=0$, one gets that

$$
\begin{aligned}
& \left|\int_{2^{-J}\left(l+N_{1}\right)}^{2^{-J}\left(l+N_{2}\right)}\left(\sigma(s)-\bar{\sigma}_{J, l}\right) \varphi\left(2^{J} s-l\right) \mathrm{d} X(s)\right| \\
& \leq\left(N_{2}-N_{1}\right)^{\alpha+\beta} \mathcal{K}_{\alpha+\beta} 2^{-J(\alpha+\beta)} \\
& \quad \times\left\|\left(\sigma(\bullet)-\bar{\sigma}_{J, l}\right) \varphi\left(2^{J} \bullet-l\right)\right\|_{C^{\alpha}\left(\mathcal{I}_{J, l}\right)}\|X\|_{C^{\beta}\left(\mathcal{I}_{J, l}\right)} \\
& \leq c_{7} 2^{-J(\alpha+\beta)},
\end{aligned}
$$

where $c_{7}=c_{6}\left(N_{2}-N_{1}\right)^{\alpha+\beta} \mathcal{K}_{\alpha+\beta}$. This shows that (2.21) holds.

Finally, let us turn to $\left(\mathcal{P}_{3}\right)$. Assume $t_{1}, t_{2} \in I$ are arbitrary and such that $t_{1}<t_{2}$. Also assume that $l \in \partial \mathbb{L}_{J, t_{1}, t_{2}}($ see $(2.17))$ and that $\mu_{J, l}=\mu_{J, l}\left(t_{1}, t_{2}\right)$ and $\nu_{J, l}=\nu_{J, l}\left(t_{1}, t_{2}\right)$ are as in (2.18). Then, one has

$$
\int_{t_{1}}^{t_{2}} \sigma(s) \varphi\left(2^{J} s-l\right) \mathrm{d} X(s)=\int_{\mu_{J, l}}^{\nu_{J, l}} \sigma(s) \varphi\left(2^{J} s-l\right) \mathrm{d} X(s) .
$$

Moreover, one can derive from the triangle inequality that

$$
\begin{aligned}
& \quad\left|\int_{\mu_{J, l}}^{\nu_{J, l}} \sigma(s) \varphi\left(2^{J} s-l\right) \mathrm{d} X(s)\right| \\
& \leq\left|\int_{\mu_{J, l}}^{\nu_{J, l}} \sigma(s) \varphi\left(2^{J} s-l\right) \mathrm{d} X(s)-\sigma\left(\mu_{J, l}\right) \varphi\left(2^{J} \mu_{J, l}-l\right)\left(X\left(\nu_{J, l}\right)-X\left(\mu_{J, l}\right)\right)\right| \\
& \quad+\left|\sigma\left(\mu_{J, l}\right) \varphi\left(2^{J} \mu_{J, l}-l\right)\left(X\left(\nu_{J, l}\right)-X\left(\mu_{J, l}\right)\right)\right|
\end{aligned}
$$

Let us now provide an appropriate upper bound for each term in the last sum. On one hand, using Proposition 1.1, (5.37) and (2.18), one obtains that

$$
\begin{aligned}
& \left|\int_{\mu_{J, l}}^{\nu_{J, l}} \sigma(s) \varphi\left(2^{J} s-l\right) \mathrm{d} X(s)-\sigma\left(\mu_{J, l}\right) \varphi\left(2^{J} \mu_{J, l}-l\right)\left(X\left(\nu_{J, l}\right)-X\left(\mu_{J, l}\right)\right)\right| \\
& \leq c_{8}\left\|\sigma(\bullet) \varphi\left(2^{J} \bullet-l\right)\right\|_{C^{\alpha}\left(\mathcal{I}_{J, l}\right)}\left(\min \left(2^{-J},\left|t_{1}-t_{2}\right|\right)\right)^{\alpha+\beta}
\end{aligned}
$$


where $c_{8}>0$ is a finite random constant not depending on $J, t_{1}$ and $t_{2}$. Moreover, one can derive from (1.2), (1.3) and the triangle inequality that

$$
\begin{aligned}
& \left\|\sigma(\bullet) \varphi\left(2^{J} \bullet-l\right)\right\|_{C^{\alpha}\left(\mathcal{I}_{J, l}\right)} \\
& \leq\|\sigma\|_{\left[Q_{1}, Q_{2}\right], \infty}\|\varphi\|_{\left[N_{1}, N_{2}\right], \infty}+\|\sigma\|_{\left[Q_{1}, Q_{2}\right], \infty}\left\|\varphi\left(2^{J} \bullet-l\right)\right\|_{C^{\alpha}\left(\mathcal{I}_{J, l}\right)} \\
& \quad+\|\sigma\|_{C^{\alpha}\left(\left[Q_{1}, Q_{2}\right]\right)}\|\varphi\|_{\left[N_{1}, N_{2}\right], \infty} \\
& \leq c_{9} 2^{\alpha J}
\end{aligned}
$$

where $c_{9}>0$ is a finite random constant not depending on $J, l, t_{1}$ and $t_{2}$. On the other hand, using the fact that the paths of $X$ are $\beta$-Hölder continuous functions on $I,(1.3)$ and (2.18), one gets that

$$
\left|\sigma\left(\mu_{J, l}\right) \varphi\left(2^{J} \mu_{J, l}-l\right)\left(X\left(\nu_{J, l}\right)-X\left(\mu_{J, l}\right)\right)\right| \leq c_{10} \min \left(2^{-J \beta},\left|t_{1}-t_{2}\right|^{\beta}\right),
$$

where $c_{10}:=\left(N_{2}-N_{1}\right)^{\beta}\|\sigma\|_{\left[Q_{1}, Q_{2}\right], \infty}\|\varphi\|_{\left[N_{1}, N_{2}\right], \infty}\|X\|_{C^{\beta}\left(\left[Q_{1}, Q_{2}\right]\right)}$. Next, putting together (5.41) - (5.45), one obtains that

$$
\begin{aligned}
\mid \int_{t_{1}}^{t_{2}} \sigma(s) & \varphi\left(2^{J} s-l\right) \mathrm{d} X(s) \mid \\
\leq & c_{11}\left(\min \left(2^{-\beta J},\left|t_{1}-t_{2}\right|^{\alpha+\beta} 2^{\alpha J}\right)+\min \left(2^{-J \beta},\left|t_{1}-t_{2}\right|^{\beta}\right)\right),
\end{aligned}
$$

where $c_{11}=\max \left(c_{8} c_{9}, c_{10}\right)$. Moreover, studying separately the two cases $t_{2}-t_{1}<$ $2^{-J}$ and $t_{2}-t_{1} \geq 2^{-J}$, one can easily show that

$$
\min \left(2^{-\beta J},\left|t_{1}-t_{2}\right|^{\alpha+\beta} 2^{\alpha J}\right) \leq \min \left(2^{-J \beta},\left|t_{1}-t_{2}\right|^{\beta}\right) .
$$

Finally combining (5.46) and (5.47) leads to (2.23).

\section{Acknowledgements}

The authors are very grateful to the anonymous associate editor and reviewer for careful reading of the paper and constructive comments.

\section{References}

F. Baudoin and L. Coutin. Operators associated with a stochastic differential equation driven by fractional Brownian motions. Stochastic Process. Appl. 117 (5), 550-574 (2007). MR2320949.

Z. Ciesielski, G. Kerkyacharian and B. Roynette. Quelques espaces fonctionnels associés à des processus gaussiens. Studia Math. 107 (2), 171-204 (1993). MR1244574.

I. Daubechies. Ten lectures on wavelets, volume 61 of CBMS-NSF Regional Conference Series in Applied Mathematics. Society for Industrial and Applied Mathematics (SIAM), Philadelphia, PA (1992). ISBN 0-89871-274-2. MR1162107.

L. Decreusefond and A. S. Üstünel. Stochastic analysis of the fractional Brownian motion. Potential Anal. 10 (2), 177-214 (1999). MR1677455.

M. Gubinelli, P. Imkeller and N. Perkowski. A Fourier analytic approach to pathwise stochastic integration. Electron. J. Probab. 21, Paper No. 2, 37 (2016). MR3485344.

M. Gubinelli, A. Lejay and S. Tindel. Young integrals and SPDEs. Potential Anal. 25 (4), 307-326 (2006). MR2255351. 
S. Janson. Gaussian Hilbert spaces, volume 129 of Cambridge Tracts in Mathematics. Cambridge University Press, Cambridge (1997). ISBN 0-521-56128-0. MR1474726.

I. Karatzas and S. E. Shreve. Brownian motion and stochastic calculus, volume 113 of Graduate Texts in Mathematics. Springer-Verlag, New York (1988). ISBN 0-387-96535-1. MR917065.

A. Lejay. Controlled differential equations as Young integrals: a simple approach. J. Differential Equations 249 (8), 1777-1798 (2010). MR2679003.

T. J. Lyons, M. Caruana and T. Lévy. Differential equations driven by rough paths, volume 1908 of Lecture Notes in Mathematics. Springer, Berlin (2007). ISBN 978-3-540-71284-8; 3-540-71284-4. MR2314753.

Y. Meyer. Wavelets and operators, volume 37 of Cambridge Studies in Advanced Mathematics. Cambridge University Press, Cambridge (1992). ISBN 0-521-420008; 0-521-45869-2. MR1228209.

D. Nualart. The Malliavin calculus and related topics. Probability and its Applications (New York). Springer-Verlag, New York (1995). ISBN 0-387-94432-X. MR1344217.

D. Nualart and A. Răşcanu. Differential equations driven by fractional Brownian motion. Collect. Math. 53 (1), 55-81 (2002). MR1893308.

A. A. Ruzmaikina. Stieltjes integrals of Hölder continuous functions with applications to fractional Brownian motion. J. Statist. Phys. 100 (5-6), 1049-1069 (2000). MR1798553.

P. Wojtaszczyk. A mathematical introduction to wavelets, volume 37 of London Mathematical Society Student Texts. Cambridge University Press, Cambridge (1997). ISBN 0-521-57020-4; 0-521-57894-9. MR1436437.

M. Zähle. Integration with respect to fractal functions and stochastic calculus. I. Probab. Theory Related Fields 111 (3), 333-374 (1998). MR1640795.

M. Zähle. Integration with respect to fractal functions and stochastic calculus. II. Math. Nachr. 225, 145-183 (2001). MR1827093. 